\title{
Detrimental and protective action of microglial extracellular vesicles on myelin lesions: astrocyte involvement in remyelination failure
}

\author{
Marta Lombardi ${ }^{1,2} \cdot$ Roberta Parolisi $^{3} \cdot$ Federica Scaroni $^{2} \cdot$ Elisabetta Bonfanti $^{4} \cdot$ Alice Gualerzi $^{5}$. \\ Martina Gabrielli ${ }^{2}$. Nicole Kerlero de Rosbo ${ }^{6}$. Antonio Uccelli ${ }^{6,7}$. Paola Giussani ${ }^{8}$. Paola Viani ${ }^{8} \cdot$ Cecilia Garlanda $^{9}$. \\ Maria P. Abbracchio ${ }^{4} \cdot$ Linda Chaabane $^{10} \cdot$ Annalisa Buffo $^{3} \cdot$ Marta Fumagalli $^{4}$. Claudia Verderio ${ }^{2}$
}

Received: 13 May 2019 / Revised: 23 July 2019 / Accepted: 24 July 2019 / Published online: 30 July 2019

(c) The Author(s) 2019

\begin{abstract}
Microglia are highly plastic immune cells which exist in a continuum of activation states. By shaping the function of oligodendrocyte precursor cells (OPCs), the brain cells which differentiate to myelin-forming cells, microglia participate in both myelin injury and remyelination during multiple sclerosis. However, the mode(s) of action of microglia in supporting or inhibiting myelin repair is still largely unclear. Here, we analysed the effects of extracellular vesicles (EVs) produced in vitro by either pro-inflammatory or pro-regenerative microglia on OPCs at demyelinated lesions caused by lysolecithin injection in the mouse corpus callosum. Immunolabelling for myelin proteins and electron microscopy showed that EVs released by pro-inflammatory microglia blocked remyelination, whereas EVs produced by microglia co-cultured with immunosuppressive mesenchymal stem cells promoted OPC recruitment and myelin repair. The molecular mechanisms responsible for the harmful and beneficial EV actions were dissected in primary OPC cultures. By exposing OPCs, cultured either alone or with astrocytes, to inflammatory EVs, we observed a blockade of OPC maturation only in the presence of astrocytes, implicating these cells in remyelination failure. Biochemical fractionation revealed that astrocytes may be converted into harmful cells by the inflammatory EV cargo, as indicated by immunohistochemical and qPCR analyses, whereas surface lipid components of EVs promote OPC migration and/or differentiation, linking EV lipids to myelin repair. Although the mechanisms through which the lipid species enhance OPC maturation still remain to be fully defined, we provide the first demonstration that vesicular sphingosine 1 phosphate stimulates OPC migration, the first fundamental step in myelin repair. From this study, microglial EVs emerge as multimodal and multitarget signalling mediators able to influence both OPCs and astrocytes around myelin lesions, which may be exploited to develop novel approaches for myelin repair not only in multiple sclerosis, but also in neurological and neuropsychiatric diseases characterized by demyelination.
\end{abstract}

Keywords Microglia $\cdot$ Extracellular vesicles $\cdot$ Mesenchymal stem cells $\cdot$ Myelin lesion $\cdot$ S1P $\cdot$ Astrocytes

$\begin{array}{ll}\text { Abbreviations } \\ \text { CC } & \text { Corpus callosum } \\ \text { cryo-EM } & \text { Cryo-electron microscopy } \\ \text { dpl } & \text { Days post lesion } \\ \text { DTI } & \text { Diffusion tensor imaging } \\ \text { DRG } & \text { Dorsal root ganglion } \\ \text { ETN } & \text { Etarnecept } \\ \text { EVs } & \text { Extracellular vesicles }\end{array}$

Electronic supplementary material The online version of this article (https://doi.org/10.1007/s00401-019-02049-1) contains supplementary material, which is available to authorized users.

Claudia Verderio

c.verderio@in.cnr.it

Extended author information available on the last page of the article

$\begin{array}{ll}\text { i-EVs } & \text { EVs from inflammatory microglia } \\ \text { IL4-EVs } & \text { EVs derived from IL4-treated microglia } \\ \text { MSC-EVs } & \text { EVs derived from MSC-treated microglia } \\ \text { ICC } & \text { Immunofluorescence } \\ \text { IHC } & \text { Immunohistochemistry } \\ \text { i-microglia } & \text { Inflammatory microglia } \\ \text { LPC } & \text { Lysolecithin } \\ \text { MSCs } & \text { Mesenchymal stem cells } \\ \text { MS } & \text { Multiple sclerosis } \\ \text { OPCs } & \text { Oligodendrocyte precursor cells } \\ \text { MACS } & \text { Magnetic-activated cell sorting } \\ \text { MRI } & \text { Magnetic resonance imaging } \\ \text { WB } & \text { Western-blot }\end{array}$




\section{Introduction}

Multiple sclerosis (MS) is a chronic demyelinating disease of the central nervous system characterized by secondary decline in myelin repair, loss of neurons and progressive disability $[3,28]$. At early disease stages, successful remyelination is sustained by oligodendrocyte precursor cells (OPCs) [33]. In response to myelin damage, these cells, also called NG2-glia, proliferate and migrate to lesion sites, where they mature to new myelin-forming cells $[64,69]$ leading to restoration of rapid neurotransmission and neurological remission. During disease chronicization up to progressive MS, remyelination is gradually impaired, primarily not only due to the inability of OPCs to differentiate into myelinating cells, but also resulting from inefficient OPC activation and recruitment to myelin lesions [60]. At the cellular/molecular levels, the interplay between OPCs and microglia and other glial cells seems to play a key role in remyelination failure [54].

Microglia are the self-renewing, long-lived brain immune cells [35] whose tasks are mainly related to immune response and homeostasis. Guided by local environmental signals [57], microglia acquire a variety of activation states, ranging from protective $[14,72]$ to harmful [37] phenotypes participating in mechanisms of tissue repair as well as to injury. More than $80 \%$ of MSspecific genes identified at actively demyelinating lesions are related to microglia activation and $\mathrm{T}$ cell-mediated inflammation [66]. Moreover, interference with microglia activation limits disease progression [37], supporting a strong relationship between microglia activation and demyelination $[28,60]$. Importantly, the negative impact of microglia on oligodendrocytes may occur through astrocytes, recognised players in MS immunopathology [12, 21], which are transformed to harmful A1 cells by inflammatory microglial mediators released from microglia (IL$1 \alpha, \mathrm{TNF}$ and C1q) [55]. On the other hand, microglial gene expression patterns suggest favourable microglial function, such as phagocytosis of myelin debris and release of growth factors in acute lesions and at the active edges of chronic active lesions where remyelination often takes place [64, 69, 97] (reviewed in [18]) [57]. In addition, in the absence of pro-regenerative microglia, OPC differentiation becomes less efficient, eventually resulting in impaired myelin repair [64, 87], whereas a switch of microglia to an anti-inflammatory phenotype promotes remyelination and ameliorates the clinical signs of experimental autoimmune encephalomyelitis (EAE) [100], an animal model of MS. Despite the demonstration of both detrimental and beneficial effects of microglia on myelin lesion, the mode(s) of action of these cells in aiding or inhibiting remyelination is still largely unclear.
Extracellular vesicles (EVs), including ectosomes/ microvesicles shed from the plasma membrane and exosomes originating from multivesicular bodies, have recently raised large interest as powerful mediators of intercellular communication between microglia and brain cells $[71,74,90]$. Production of microglial EVs dramatically increases under brain inflammation [22, 27, 61, 95] reflecting the extent of microglia activation. Following interaction with the recipient cells, microglia-derived EVs activate contact-mediated signaling pathways $[1,34]$ and/or deliver genetic information [25, 75, 95], thus profoundly influencing the molecular configuration and function of the target cells. Specifically, microglia-derived EVs propagate an activation signal to astrocytes and to other microglia $[25,95]$ and enhance excitatory neurotransmission [1]. However, whether microglial EVs play a role in the cross-talk between microglia and OPCs or not is still unknown.

Here, we have investigated the effects on OPCs of EVs released in vitro from either pro-inflammatory or proregenerative microglia both in vivo in the lysolecithin (LPC) mouse model of focal demyelination and in vitro in primary OPC cultures. Although classification in inflammatory and pro-regenerative cells underestimates the complexity of microglial activation, such a definition provides a useful framework for investigating the impact of microglia on OPCs. To induce pro-regenerative traits, microglia were exposed to the classical polarising agent IL-4 or to immunosuppressive mesenchymal stem cells (MSCs) [36, 101], while inflammatory state was obtained through stimulation with inflammatory cytokines.

We found that EVs produced by inflammatory microglia led to blockade of OPC differentiation to myelin-forming cells. Conversely, EVs released from microglia exposed to MSCs promoted remyelination. Biochemical fractionation combined with in vitro experiments revealed a major contribution of the inflammatory cargo of EVs to blocking OPC maturation, while surface lipids were identified as responsible factors for the pro-myelinating action of EVs. Although the type of lipids driving OPC differentiation still remains to be fully defined, sphingolipid sphingosine 1 phosphate (S1P) was unveiled as the key molecule favouring OPC migration, the first fundamental step in the remyelination process.

\section{Materials and methods}

\section{Lysolecithin-induced myelin lesions}

Demyelinating lesions were induced in the corpus callosum (CC) of adult (2.5-4 months old) and aged (8-12 months old) male C57BL/6 mice by stereotaxic unilateral injection of $1 \mu \mathrm{l}$ of $2 \%$ lysolecithinto the mouse corpus callosum (CC, coordinates: $1.0 \mathrm{~mm}$ lateral, $1.0 \mathrm{~mm}$ rostral to Bregma and 
$1.5 \mathrm{~mm}$ deep). Three days post lesion (dpl), $2 \times 10^{8} \mathrm{EVs}$, produced by $1.5 \times 10^{6}$ microglia and dissolved in $150 \mu$ of sterile saline, were delivered to the mice with mini-pumps (Alzet osmotic pumps 1007D) at the same coordinates over 4 days at $1.5 \mu \mathrm{h}^{-1}$ delivery rate. To limit EV degradation, minipumps were filled with freshly isolated EVs and implanted within a few hours. Controls were obtained with delivery of saline solution. To examine cell proliferation, we used the thymidine analog 5-bromo-2-deoxyuridine (BrdU), which is incorporated into DNA during S-phase of the cell cycle and remains in the DNA even when the cell has exited the active phases of the cell cycle. Mice were i.p. injected with BrdU (100 mg kg ${ }^{-1}$ body weight; 2 pulses $2 \mathrm{~h}$ apart) 1 day from the start of EVs delivery. A separate group of mice received a single injection of $6.6 \times 10^{7} \mathrm{EVs}$, released from $0.5 \times 10^{6}$ microglia and dissolved in $1 \mu \mathrm{l}$ of sterile saline, at the site of lesion at $7 \mathrm{dpl}$. Controls were obtained by injecting saline. Mice were transcardially perfused with $4 \%$ paraformaldehyde in phosphate buffer $(\mathrm{PB} 0.1 \mathrm{M}, \mathrm{pH}$ 7.4) at 4 days after mini-pump implantation or at 3 days after acute injection and brains were post-fixed overnight and cryoprotected in $30 \%$ sucrose in 0.12 M PB. Surgery and perfusion were carried out under deep general anaesthesia (ketamine, $100 \mathrm{mg} \mathrm{kg}^{-1}$, Ketavet, Bayern, Leverkusen, Germany; xylazine, $5 \mathrm{mg} \mathrm{kg}^{-1}$, Rompun, Bayer, Leverkusen, Germany). All animal procedures were performed in accordance with the European directive (2010/63/EU) and the Italian Law for Care and Use of Experimental Animals (DL116/92; DL26/2014), and the studies were authorised by the Italian Ministry of Health (Authorization: 1112-2016PR) and the Bioethical Committee of the University of Turin.

\section{Electron microscopy of myelin lesions}

Conventional electron microscopy was carried out as in [5]. LPC-lesioned old mice injected with saline, i-EVs or MSC-EVs were perfused transcardially with PB followed by $2 \%$ paraformaldehyde and $2.5 \%$ glutaraldehyde in PB. The brain was removed and post-fixed overnight at $4{ }^{\circ} \mathrm{C}$ in the same fixative. Vibratome sagittal sections $(250 \mu \mathrm{m}$ thick $)$ were cut, and post-fixed with $1 \%$ osmium tetroxide for $1 \mathrm{~h}$ at $4{ }^{\circ} \mathrm{C}$, then stained with uranyl acetate replacement stain (Electron Microscopy Sciences, USA). After dehydration in ethanol, samples were cleared in propylene oxide and embedded in Araldite (Fluka, Saint Louis, USA). Semithin sections $(1 \mu \mathrm{m}$ thick) were obtained at the ultramicrotome (Ultracut UCT, Leica, Wetzlar, Germany), stained with $1 \%$ toluidine blue and $2 \%$ borate in distilled water and then observed under a light microscope for precise lesion location. Ultrathin sections $(70-100 \mathrm{~nm})$ were examined under a transmission electron microscope (JEOL, JEM-1010, Tokyo, Japan) equipped with a Mega-View-III digital camera and a Soft-Imaging-System (SIS, Münster, Germany) for computerized acquisition of the images. Profiles of microglia, astrocytes, oligodendrocytes and myelinated axons were identified according to well established criteria [78]. Microglia were distinguished by their specific ultrastructural features including frequent stretches of endoplasmic reticulum and a condensed, electron-dense cytoplasm [7, 89]. In the core of the lesion, electron micrographs were taken at $25 \mathrm{~K}$ magnification and ImageJ software (https:// imagej.nih.gov/ij/) was used to measure the proportion of myelinated axons (on at least 150 axons/animal) and perform the measurements needed to obtain G-ratios, the ratios of the axonal diameter (d white, Fig. 4k) to the outer fibre diameter including the myelin sheath (d yellow, Fig. 4k), of at least 50 myelinated axons/animal. Quantifications were performed on three mice (8-12 months old) per experimental condition.

\section{Immunohistochemistry}

Brains were processed according to standard immunohistochemical procedures [9]. They were cut into $30 \mu \mathrm{m}$-thick coronal sections, collected in PBS and stained with the antibodies reported in the Table 1. OPCs were labelled by anti-NG2 antibodies while more mature cells were detected by anti-CC1 serum. AntiOlig2 and anti-Sox 10 stained the whole oligodendrocyte lineage. Anti-MBP antibodies labelled myelin. To follow cell proliferation, we used anti-BrdU. DAPI or Hoechst33258 were used to label nuclei. Anti-GFAP, antiPTX3 and anti-C3 were used to label astrocytes, Iba-1 to reveal microglia. Sections were incubated overnight with primary antibodies at $4{ }^{\circ} \mathrm{C}$ in PBS with $1.5 \%$ donkey serum, $2 \%$ bovine serum albumin and $0.5 \%$ Triton $\mathrm{X}-100$. Sections were then exposed for $2 \mathrm{~h}$ at room temperature to secondary anti-rabbit, anti-chicken antibodies conjugated with Alexa Fluor 488 or anti-rabbit antibodies conjugated with Alexa Fluor 546 (1:500; Invitrogen, Waltham, MA, USA), anti-mouse, anti rabbit antibodies conjugated with Cy3 (1:500; Jackson ImmunoResearch, West Grove, PA, USA), anti-goat antibodies conjugated with Alexa Fluor 649 (1:500; Invitrogen) or anti-mouse antibodies conjugated with Cy5 (1:500; Jackson ImmunoResearch). Double staining with rabbit anti-GFAP and rabbit anti-PTX3/anti-C3 was performed using the High Sensitivity Tyramide-Rhodaminate Signal Amplification kit (Perkin-Elmer, Monza, Italy) following the manufacturer's instructions. Following counterstaining with 4,6-diamidino-2-phenylindole (DAPI) (SigmaAldrich Co., St. Louis, MO, USA) or Hoechst 33258 (Life Technologies, Monza, Italy) to label nuclei, slides were coverslipped with Mowiol (Millipore, Burlington, MA, USA) or with a fluorescent mounting medium from Dako (Milan, Italy). All images were collected using a Nikon 
Table 1 List of primary antibodies used for immunohistochemistry (IHC), immunofluorescence (ICC) and western-blot (WB) analyses

\begin{tabular}{|c|c|c|c|c|c|}
\hline Antibody & Host & Supplier & IHC dilution & ICC dilution & $\overline{\text { WB dilution }}$ \\
\hline Anti-NG2 & Rabbit & Millipore (Burlington, MA, USA) & $1: 200$ & $1: 100$ & \\
\hline Anti-CC1 & Mouse & Millipore (Burlington, MA, USA) & $1: 1500$ & & \\
\hline Anti-CNPase & Rabbit & SantaCruz (Dallas, Texas) & & & $1: 250$ \\
\hline Anti-Olig2 & Rabbit & Millipore (Burlington, MA, USA) & $1: 500$ & $1: 300$ & \\
\hline Anti-SOX-10 & Goat & SantaCruz (Dallas, Texas) & $1: 200$ & & \\
\hline Anti-MBP & Rat & AbD Serotec (Oxford, UK) & & $1: 200$ & \\
\hline Anti-MBP & Rat & Covance (Princeton, $\mathrm{NJ}$ ) & $1: 1000$ & & \\
\hline Anti-MBP & Rat & Millipore (Burlington, MA, USA) & & $1: 200$ & $1: 500$ \\
\hline Anti-BrdU & Rat & Abcam (Cambridge, UK) & $1: 500$ & & \\
\hline Anti-pentraxin 3 & Rabbit & $\begin{array}{l}\text { Home-made generated as previously described } \\
{[10]}\end{array}$ & $1: 250$ & & \\
\hline Anti-C3 & Rabbit & Dako (Milan, Italy) & $1: 250$ & & \\
\hline Anti-GFAP & Rabbit & Dako (Milan, Italy) & $\begin{array}{l}\text { 1:30,000 with } \\
\text { amplification } \\
\text { kit }\end{array}$ & & \\
\hline Anti-GFAP & Mouse & Sigma-Aldrich (Milan, Italy) & & $1: 100$ & \\
\hline Anti- Neurofilaments (SMI32) & Mouse & Cell Signaling (Beverly, MA, USA) & & $1: 500$ & \\
\hline Anti- Neurofilaments (SMI31) & Mouse & Cell Signaling (Beverly, MA, USA) & & $1: 500$ & \\
\hline Anti- $\alpha$-tub & Mouse & Sigma-Aldrich & & & $1: 2000$ \\
\hline Anti-GSTpi & Rabbit & MBL International Corporation (Sunnyvale, CA) & & & $1: 500$ \\
\hline Anti-GPR17 & Rabbit & $\begin{array}{l}\text { Home-made generated as previously described, } \\
\text { Ciana } 2006\end{array}$ & & & $1: 1000$ \\
\hline Anti-GPR17 & Rabbit & Cayman Chemical (Michigan, USA) & & $1: 100$ & \\
\hline Anti-GFP & Chicken & AVES Labs & $1: 700$ & $1: 1400$ & \\
\hline Anti-GFP & Rabbit & Life Technologies (Monza, Italy) & & & $1: 3000$ \\
\hline Anti-Iba1 & Rabbit & Wako Chemicals (Richmond, VA) & $1: 1000$ & & \\
\hline 4',6-Diamidino-2-phenylindole DAPI & & Fluka (Buche, Switzerland) & $1: 1000$ & & \\
\hline DAPI & & $\begin{array}{l}\text { Molecular Probes (Life Technologies, Monza, } \\
\text { Italy) }\end{array}$ & & $1: 20.000$ & \\
\hline Hoechst 33258 & & Life Technologies (Monza, Italy) & $1: 50,000$ & $1: 10,000$ & \\
\hline Anti-Alix & Rabbit & Covalab (Villeurbanne, France) & & & $1: 500$ \\
\hline Anti-Flotilin 2 & Mouse & BD transduction (San Jose, CA) & & & $1: 1000$ \\
\hline Anti-GAPDH & Rabbit & Synaptic Systems (Goettingen, Germany) & & & $1: 1000$ \\
\hline Anti-GS28 & Mouse & BD transduction (San Jose, CA) & & & $1: 1000$ \\
\hline
\end{tabular}

Eclipse 90i confocal microscope (Nikon, Melville, NY, USA), or using a Zeiss LSM 800 confocal microscope (Carl Zeiss S.p.A, Milano, Italy). Adobe Photoshop 6.0 (Adobe Systems) was used to adjust image contrast and assemble the final plates. Measurements were derived from at least three sections per mice. Three to five animals were analysed per each time point or experimental condition. Image analysis was performed with the Image J software to quantify the proportion of NG2 or MBPstained pixels throughout the entire lesioned area in each section [46, 53, 64]. Density of oligodendroglial, proliferative cells and PTX3- and C3-positive astrocytes in the lesion was calculated as number of cells $\mathrm{mm}^{-2}$, using the Image J software.

\section{Magnetic resonance imaging (MRI)}

In vivo MRI was performed using a $7 \mathrm{~T}$ scanner (BrukerBiospin) equipped with a radiofrequency coil for mouse. Mice were anaesthetized with an intramuscular injection of Ketamine (100 mg kg-1; Ketavet, Bayern, Leverkusen, Germany) mixed with xylazine $\left(5 \mathrm{mg} \mathrm{kg}^{-1}\right.$; Rompun, Bayer, Leverkusen, Germany). High Resolution coronal and sagittal images, T2 weighted (TR/TE $=3000 / 48 \mathrm{~ms}$, slice thickness $=0.7 \mathrm{~mm}$, pixel $=78 \times 80 \mu \mathrm{m}$ ) were acquired to localise lesions in the corpus callosum. Diffusion tensor imaging (DTI: 30 directions, $b=1000 \mathrm{~s} \mathrm{~mm}^{-2}$ ) was also used to measure water molecule diffusivity across white matter fibres to further characterise CC lesions. 


\section{Primary cultures}

Mixed glial cell cultures, containing both astrocytes and microglia, were established from rat Sprague-Dawley pups (P2) (Charles River, Lecco, Italy) and maintained for 10 days in the presence of South American foetal bovine serum (Life Technologies, Monza, Italy) that optimises microglia expansion or foetal bovine serum (EuroClone, Milan, Italy) which favours OPC proliferation.

\section{Microglia}

Microglia were harvested by orbital shaking for $40 \mathrm{~min}$ at 1300 r.p.m. and re-plated on poly-L-ornithine-coated tissue culture dishes $\left(50 \mu \mathrm{g} \mathrm{ml}^{-1}\right.$, Sigma-Aldrich, Milan, Italy). To minimise the activation, pure microglia ( $>98 \%$, [75] were kept for $24 \mathrm{~h}$ in low-serum (1\%) medium. Cells were then stimulated with a cocktail of Th1 cytokines, i.e. $20 \mathrm{ng} \mathrm{ml}^{-1}$ IL-1 $\beta$ (Peprotech, Milan, Italy), $20 \mathrm{ng} \mathrm{ml}^{-1}$ TNF- $\alpha$ (Peprotech, Milan, Italy) and $25 \mathrm{ng} \mathrm{ml}^{-1}$ IFN-y (Sigma Aldrich, Milan, Italy), or with $20 \mathrm{ng} \mathrm{ml}^{-1} \mathrm{IL}-4$ for $48 \mathrm{~h}$ (R\&D, Milan, Italy). In addition, microglia were co-cultured in transwell with MSCs at a microglia-to-MSCs ratio of 1:1 for $48 \mathrm{~h}$ in the presence of Th1 cytokines. MSCs were plated on the filter of the upper chamber and microglia in the lower chamber of the transwell. At the end of treatment, MSCs were removed, microglia were washed and stimulated with ATP to increase EV production [75]. To obtain GFP-labelled EVs, microglia were established from GFP-transgenic rats expressing GFP under the chicken beta actin promoter [68]. Indeed, GFP is included in EVs released by GFP-positive microglia (Suppl. Figure 3h, Online resource).

\section{OPCs}

After microglia removal, OPCs growing on top of astrocyte monolayer were isolated by shaking cells on an orbital shaker at $200 \mathrm{rpm}$ for $3 \mathrm{~h}$ and incubated on an uncoated Petri dish for $1 \mathrm{~h}$ to further eliminate microglia. Pure OPCs (>95\% [32] were seeded onto poly-D,L-ornithine-coated glass coverslips or plates $\left(50 \mu \mathrm{g} \mathrm{ml}^{-1}\right.$, Sigma-Aldrich, Milan, Italy) in Neurobasal (Life Technologies, Monza, Italy) supplemented with 2\% B27 (Life Technologies, Monza, Italy), $2 \mathrm{mM}$ L-glutamine (EuroClone, Milan, Italy), $10 \mathrm{ng} \mathrm{ml}^{-1}$ human platelet-derived growth factor BB (Sigma-Aldrich, Milan, Italy), and $10 \mathrm{ng} \mathrm{ml}^{-1}$ human basic fibroblast growth factor (Space Import Export, Milan, Italy), to promote proliferation (proliferating medium). After 3 days, cells were either detached with accutase (Millipore, Burlington, MA, USA) and used for migration assay, or switched to a Neurobasal medium lacking growth factors and supplemented with triiodothyronine T3 $\left(10 \mathrm{ng} \mathrm{ml}^{-1}\right.$, Sigma Aldrich, Milan, Italy) to allow differentiation (differentiating medium).

\section{Dorsal root ganglion (DRG)-OPC co-cultures}

DRG-OPC co-cultures were prepared according to a previously described protocol [31]. Briefly, DRG from E14.5 mouse embryos were plucked off from spinal cord, put in culture (1 DRG/coverslip) in Neurobasal supplemented with B27 in the presence of nerve growth factor (NGF) (100 $\mathrm{ng} \mathrm{ml}^{-1}$; Harlan, Milan, Italy) and cycled with fluorodeoxyuridine ( $10 \mu \mathrm{M}$; Sigma Aldrich, Milan, Italy) to eliminate all non-neuronal cells. After 20 days, when neurites were well extended radially from DRG explants, $35 \times 10^{3}$ OPCs were added to each DRG in culture and kept in Minimum essential media MEM (Life Technologies Monza, Italy) supplemented with glucose $\left(4 \mathrm{~g}^{-1}\right.$; Sigma Aldrich, Milan, Italy), 10\% FBS and $2 \mathrm{mM}$ L-glutamine (EuroClone, Milan, Italy). Myelination was induced the following day by the addition of recombinant chimeric tyrosine kinase receptor TrkA Fc $\left(1 \mu \mathrm{g} \mathrm{m}{ }^{-1}\right.$; Sigma Aldrich, Milan, Italy) to the culture medium.

\section{Astrocyte-OPC co-cultures}

Purified astrocytes were isolated from $\mathrm{P} 2$ rat whole brains by magnetic-activated cell sorting (MACS) (Miltenyi Biotec, Bergisch Gladbach, Germany) with anti-GLAST (ACSA 1) MicroBeads according to the manufacturer's instructions. After 1 week, OPCs were plated on top of astrocytes at an astrocyte-to-OPC ratio of 1:1. In a set of experiments astrocytes were isolated from $\mathrm{P} 2$ mouse whole brains and cultured alone for qPCR analysis of specific markers for $\mathrm{A} 1$ and $\mathrm{A} 2$ astrocytes.

\section{MSCs}

MSCs were prepared and expanded as described previously [102]. Briefly, marrow cells were flushed out from tibias and femurs of 6- to 8-week-old C57BL/6J mice and cultured in plastic plates as adherent cells using murine Mesencult as medium (Stem Cell Technologies, Vancouver, BC, Canada). Medium was refreshed every 3 days until cells reached $80 \%$ confluence. Following treatment with $0.05 \%$ trypsin solution containing $0.02 \%$ EDTA (Euroclone, Milan, Italy), the cells were plated in $75 \mathrm{~cm}^{2}$ flask at the density of $4 \times 10^{5}$ cells. Mature MSCs, obtained after four to five passages in culture, were defined by the expression of CD9, Sca-1, CD73, and CD44 and the lack of the hematopoietic markers CD45, CD34, and $\mathrm{CD} 11 \mathrm{~b}$ on their surface, and their immunosuppressive activity was verified in $\mathrm{T}$ cell proliferation assays [102]. 


\section{RNA isolation and qRT-PCR}

Total RNA was isolated from rat primary microglia using Direct-zol $^{\mathrm{TM}}$ RNA MiniPrep (Zymo Research, Irvine, CA, USA) following the manufacturer's protocol. cDNA synthesis was performed using High Capacity cDNA Reverse Transcription Kit (Applied Biosystems, Foster City, CA, USA) and Random Hexamers as primer. The resulting cDNAs were amplified using TaqMan ${ }^{\circledR}$ Gene Expression Assay (Applied Biosystems, Foster City, CA, USA) using QuantStudio $^{\text {TM}} 5$ (ThermoFisher Scientific, Waltham, MA, USA) real-time PCR system. The mRNA expression was normalised to the label of Rpl13 (Ribosomal Protein L13) mRNA. Data obtained were quantified using the $2^{-\Delta \Delta C T}$ method [56]. Q-PCR for A1 and A2 markers was performed on murine astrocytes with the Luna Universal Probe qPCR Master Mix (M3004S, New England Biolabs) using the StepOne ${ }^{\mathrm{TM}}$ Plus Real-Time PCR System (Life Technologies, Monza, Italy). The expression of selected genes was normalized to the expression of the housekeeping gene $\beta$-actin. The list of primers used can be found in Table 2 .

\section{EV isolation and quantification}

EVs were isolated from microglia exposed to Th1 cytokine cocktail (i-EVs), IL-4 (IL4-EVs) or MSC (MSC-EVs). To isolate EVs, polarised microglia were stimulated with $1 \mathrm{mM}$ ATP for $30 \mathrm{~min}$ in $\mathrm{KRH}(125 \mathrm{mM} \mathrm{NaCl}, 5 \mathrm{mM} \mathrm{KCl}, 1.2 \mathrm{mM}$

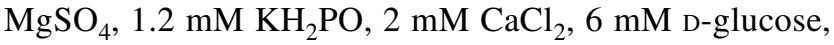
and $25 \mathrm{mM}$ HEPES/NaOH, $\mathrm{pH}$ 7.4). The culture supernatant was collected and released EVs were pelleted at $100 \mathrm{~kg}$ after pre-clearing from cells and debris at $300 \mathrm{~g}$, as previously described [34]. In some experiments, an ectosome-enriched fraction was pelleted at $10 \mathrm{~kg}$. EV pellets were resuspended and used immediately after isolation. The number and dimension of EVs were assessed using NanoSight NS300 (NanoSight, UK) configured with a 488-nm laser and SCMOS camera. Videos were collected and analysed using the NTA-software (version 2.3), with the minimal expected particle size, minimum track length, and blue setting, all set to automatic. Camera shutter speed was fixed at $15 \mathrm{~ms}$ and camera gain was set to 300 . Room temperature was ranging from 25 to $28^{\circ} \mathrm{C}$. EV pellets were re-suspended in $800 \mu \mathrm{l}$ of $0.1 \mu \mathrm{m}$-filtered sterile $\mathrm{KRH}$ and four recordings of $30 \mathrm{~s}$ were performed for each sample.

EVs were destroyed by hypo-osmotic stress and re-pelleted to remove their luminal cargo as previously described [34]. For biochemical fractionation of EVs, total lipids were extracted with 2:1 (by volume) of chloroform and methanol. The lipid fraction was evaporated under a nitrogen stream, dried for $1 \mathrm{~h}$ at $50{ }^{\circ} \mathrm{C}$ and resuspended in PBS at $40{ }^{\circ} \mathrm{C}$ in order to obtain multilamellar vesicles. Small unilamellar vesicles were obtained by sonicating multilamellar vesicles, following the procedure of [4].

\section{Quantification of sphingolipid content in EVs}

$\left[{ }^{3} \mathrm{H}\right]$ Sphingosine $\left(\left[{ }^{3} \mathrm{H}\right] \mathrm{Sph}\right)$ was stocked in absolute ethanol. For the quantification of sphingolipid content in EVs, $1 \times 10^{6}$ microglia were pulsed with $\left[{ }^{3} \mathrm{H}\right] \mathrm{Sph}\left(0.3 \mu \mathrm{Ci} \mathrm{ml}{ }^{-1}\right)$ for $24 \mathrm{~h}$ followed by a 48 -h chase in order to obtain a $\left[{ }^{3} \mathrm{H}\right]$ $\mathrm{GM} 3 /\left[{ }^{3} \mathrm{H}\right] \mathrm{SM}$ ratio corresponding to that of endogenous compounds (data not shown), an index for a steady-state labelling of cell sphingolipids [96]. At the end of the chase, microglia were stimulated with ATP, EVs were pelleted and the cells were washed twice with PBS at $4{ }^{\circ} \mathrm{C}$ and harvested. Total lipids were extracted from both EVs and cells and processed as previously described $[79,80]$. The organic phase and the aqueous phase were analysed by high-performance thin-layer chromatography (HPTLC) using chloroform/
Table 2 List of gene expression assays for qPCR

\begin{tabular}{lll}
\hline Gene symbol & Name & Taqman assay \\
\hline Arg 1 & Arginase 1 & Rn00691090_m1 \\
C1q & Complement C1q & Rn01519903_m1 \\
IL1-a & Interleukin 1 Alpha & Rn00566700_m1 \\
IL1- $\beta$ & Interleukin 1 Beta & Rn00580432_m1 \\
iNOS & Nitric Oxide Synthase 2 & Rn00561646_m1 \\
Rp113a & Ribosomal Protein L13 & Rn00821946_g1 \\
Socs-3 & Suppressor Of Cytokine Signaling 3 & Rn00585674_s1 \\
TNF- $\alpha$ & Tumour Necrosis Factor & Rn99999017_m1 \\
Amigo 2 & Adhesion Molecule With Ig Like Domain 2 & 04688058001 \\
$\beta-A c t i n$ & Actin Beta & 04688635001 \\
CD14 & Monocyte Differentiation Antigen CD14 & 04687574001 \\
Ptx3 & Pentraxin 3 & 04686993001 \\
Tm4sf1 & Transmembrane 4 L Six Family Member 1 & 04687990001 \\
Serping1 & Serpin Family G Member 1 & 04689011001 \\
\hline
\end{tabular}


methanol/water ( $55: 20: 3$ by volume) and butanol/acetic acid/ water (3:1:1 by volume) as the solvent systems.

\section{Raman spectroscopy}

The Raman analysis was performed following a previously described protocol [40]. Briefly, freshly isolated EVs were laid on a calcium fluoride slide and allowed to air dry. All of the measurements were performed with a Raman microspectroscope (LabRAM Aramis, Horiba Jobin-Yvon S.A.S, Lille, France) equipped with a laser line operating at $532 \mathrm{~nm}$ and a Peltier-cooled CCD detector. Acquisitions were performed with 50X objective (NA 0.75, Olympus, Tokyo, Japan), 1800 grooves $\mathrm{mm}^{-1}$ diffraction grating, $400 \mu \mathrm{m}$ entrance slit and confocal mode $(600 \mu \mathrm{m}$ pinhole $)$ in the spectral ranges $600-1800 \mathrm{~cm}^{-1}$ and $2600-3200 \mathrm{~cm}^{-1}$. Accumulation times were $2 \times 30 \mathrm{~s}$ per spectrum. Silicon reference sample was used for the instrument calibration. At least 30 independent replicates of the Raman spectra were obtained for every EV type. After acquisition, polynomial baseline correction and unit vector normalisation were performed before the multivariate statistical analysis.

\section{OPC migration assay}

Migration of OPCs was performed in Boyden chambers ( $8 \mu \mathrm{m}$ pore size filter; Constar, Corning, NY, USA) as previously described [11]. Briefly, the chamber was nested inside the well of 24-well plates and $5 \times 10^{4}$ OPCs were seeded in the top of each insert with $200 \mu \mathrm{l}$ of neurobasal medium. The bottom well was filled with $600 \mu \mathrm{l}$ of medium containing EVs released from $1 \times 10^{5}$ microglia $\left(\sim 4 \times 10^{8}\right.$ EVs ml $\left.{ }^{-1}\right)$. The chemotactic agent sphingosine-1 phosphate (S1P) $(100 \mathrm{nM})$ was used as positive control. After $16 \mathrm{~h}$, non-migrated cells were removed from the top compartment with a cotton swab, whereas cells that had migrated to the lower side of the filter were fixed with $4 \%$ paraformaldehyde and stained with Hoechst33258 (Life Technologies, Monza, Italy). Images were acquired at $20 \times$ magnification under an inverted fluorescence microscope (200 M; Zeiss, Oberkochen, Germany) and cells were counted using ImageJ cell counter plugin in 45 random fields per well. All conditions were run in triplicate. Data are expressed as a percentage of basal migration that is the migration of OPCs without chemoattractant.

\section{EV delivery by optical manipulation}

An IR laser beam (1064 nm, CW) for trapping was coupled into the optical path of an inverted microscope (Axiovert 200 M, Zeiss, Oberkochen, Germany) through the right port of the microscope. The trapping beam was directed to the microscope lens (Zeiss 63X, NA 1.4) by the corresponding port mirror (100\%) and the tube lens. Optical trapping and manipulation of EVs were performed following the approach previously described [73]. Immediately before recording, EVs were added in the temperature-controlled recording chamber, where OPCs plated on glass coverslips were maintained in $400 \mu \mathrm{l}$ of Neurobasal medium with B27. As soon as an EV appeared in the recording field, it was trapped and positioned on a selected OPC by moving the cell stage horizontally and the microscope lens axially. After about $30 \mathrm{~s}$ from initial contact, the laser was switched off to prove EVOPC interaction, as previously described [73]. During the experiments, OPCs were live-imaged with a spinning disk confocal microscope (UltraVIEW acquisition system, Perkin Elmer Waltham, MA, USA) using a digital camera (High Sensitivity USB 3.0 CMOS Camera $1280 \times 1024$ Global Shutter Monochrome Sensor, Thorlabs, Newton, NJ, USA) at a frame rate of $2 \mathrm{~Hz}$.

\section{OPC proliferation assay}

One day after plating on glass coverslips, OPCs were coexposed to EVs derived from twice as many microglia $\left(\sim 2 \times 10^{7} \mathrm{EVs} / 500 \mu \mathrm{l}\right)$ and to the thymidine analog EdU (Click-iT ${ }^{\circledR}$ EdU Assay, Life Technologies, Monza, Italy) for $24 \mathrm{~h}$ in proliferating medium. Cells were fixed with $4 \%$ paraformaldehyde and stained for EdU following the manufacturer's instructions. Coverslips were then incubated with DAPI (1:20000, Molecular Probes, Life Technologies) to reveal total nuclei and mounted with a fluorescent mounting medium (Dako, Milan, Italy). 40-50 fields per coverslip were imaged at $20 \times$ magnification under an inverted fluorescence microscope (200 M Zeiss, Oberkochen, Germany) connected to a PC computer equipped with the Axiovision software (Zeiss). OPC proliferation was assessed by quantifying EdU-DAPI double positive nuclei in at least three coverslips/condition, using ImageJ cell counter plugin. Cells with nuclei larger than $15 \mu \mathrm{m}$, belonging to astrocytes occasionally present in the cultures, were excluded from the analysis.

\section{OPC differentiation assay}

OPCs were kept for 3 days in proliferating medium, shifted to differentiating medium for $24 \mathrm{~h}$ and then exposed to EVs for $48 \mathrm{~h}\left(2 \times 10^{7} \mathrm{EVs} / 500 \mu \mathrm{l}\right)$. Cells were fixed and labelled with anti-G Protein-Coupled Receptor 17 (GPR17) and anti-MBP (Table 1) in Goat Serum Dilution Buffer (GSDB; $450 \mathrm{mM} \mathrm{NaCl}, 20 \mathrm{mM}$ sodium phosphate buffer, $\mathrm{pH}$ 7.4, $15 \%$ goat serum, $0.3 \%$ Triton $\mathrm{X}-100$ ), followed by secondary antibodies conjugated to Alexa Fluor 555 or Alexa Fluor 488 (1:200; Molecular Probes, Life Technologies). Differentiation towards mature oligodendrocytes was determined by counting $\mathrm{MBP}^{+}$cells over total DAPI+ cells in 35-45 fields 
per coverslip with ImageJ software. GPR17 staining was used to reveal immature oligodendrocytes, the most abundant oligodendrocyte population after 3 day in differentiating medium.

\section{OPC myelination assay}

OPC-DRG co-cultures were kept in $1 \mu \mathrm{g} \mathrm{ml}^{-1}$ TrkA-Fc for 5 days and then exposed to EVs for 11 days (fresh EVs, $2 \times 10^{7} \mathrm{EVs} / 500 \mu \mathrm{l}$, were added at day in vitro (DIV5, DIV8 and DIV12). Cells were fixed at DIV16 with paraformaldehyde and labelled with anti-MBP and anti-high-molecularweight neurofilaments (NF) antibodies (SMI31 and SMI32 in Table 1) in GSDB, followed by secondary antibodies conjugated to Alexa Fluor 555 or Alexa Fluor 488 (1:600; Molecular Probes, Life Technologies). Nuclei were labelled with DAPI. Coverslips were mounted with a fluorescent mounting medium (Dako, Milan, Italy). For the co-culture analysis, stacks of images of MBP- and SMI31- and SMI32positive cells were taken under confocal microscope at $40 \times$ magnification (at 6 fields/coverslip) and the ZEISS LSM Image Browser was utilised to automate quantification of the myelination index. Images in the stack were merged at each level and pixels overlapping in the red and green fields above a predefined threshold intensity value were highlighted in white. The amount of myelin per axon (myelination index) was calculated as the ratio between the white pixel and the green pixel areas.

\section{Western blot analysis}

OPCs were lysed with a buffer containing $1 \%$ sodium dodecyl sulphate (SDS), $10 \mathrm{mM}$ HEPES, $2 \mathrm{mM}$ EDTA $\mathrm{pH}$ 7.4. A modified version of the Laemmli buffer $(20 \mathrm{mM}$ Tris pH 6.8, 2 mM EDTA, 2\% SDS, 10\% glycerol, 2\% $\beta$-mercaptoethanol, $0.01 \%$ bromophenol blue) was then added to a final $1 \times$ concentration and proteins were separated by electrophoresis, blotted on nitrocellulose membrane filters and probed using the using the primary antibodies reported in the Table 1 and the HRP-conjugated secondary antibodies (goat anti-rat 1:1000, goat anti-rabbit 1:4000 and goat anti-mouse 1:2000; Sigma Aldrich, Milan, Italy). Photographic development was by chemiluminescence (ECL, GE Healthcare) according to the manufacturer's instructions. Densitometric analysis of the protein bands was performed with ImageJ software. Band intensities were measured as integrated density volumes (IDV) and expressed as percentage of control lane values.

\section{ELISA}

The concentration of IL-1a or C1q or TNF- $\alpha$ in microglial cells or EVs was quantified using a solid-phase sandwich
ELISA (enzyme-linked immunosorbent assay) kit following the manufacturer's protocol (Rat IL-1a ELISA kit, Invitrogen Waltham, MA, USA, BMS627; Rat TNF- $\alpha$ ELISA kit, Invitrogen Waltham, MA, USA, BMS622, Rat C1q ELISA kit, Novus Biologicals Centennial, CO, USA, NBP2-74988). IL-1a or C1q or TNF- $\alpha$ content in the cells was determined after cell lysis with RIPA Buffer (Sigma Aldrich, Milan, Italy), whereas inside EVs after detergent permeabilization with $0.6 \%$ Triton X-100 (Sigma Aldrich, Milan, Italy) in the presence of protease inhibitors (1:1000, Sigma Aldrich, Milan, Italy). Sample absorbance was measured with a spectrophotometric system (1420 Multilabel Counter Victor 2; Wallac) at $450 \mathrm{~nm}$ at $10 \mathrm{~Hz}$. The amount of IL-1a, C1q or TNF- $\alpha$ in EVs was estimated on the basis of a standard curve in the presence $0.6 \%$ Triton $\mathrm{X}-100$.

\section{Cryo-electron microscopy}

Cryo-EM allows imaging of samples without the addition of any heavy metals or fixatives, which might cause artefacts, with the drawback of yielding a lower contrast. The sample is frozen so rapidly that the water vitrifies forming no ordered crystals, and the native structure of the sample is preserved $[26,70]$. Freshly prepared vesicles resuspended in saline were plunge frozen in liquid propane using a Vitrobot Mark IV (ThermoFisher Scientific, Oregon, USA).

\section{Drugs and reagents}

S-FTY720-Vinylphosphonate (kind gift from Prof. Robert Bittman) was dissolved in fatty acid-free bovine serum albumin (1 mM in PBS). S1P (Enzo Biochem. Inc, Farmingdale, NY, USA) was dissolved in fatty acid-free bovine serum albumin (4 $\mathrm{mg} \mathrm{ml}^{-1}$ in distilled water). Stock solutions were diluted in fresh Krebs-Ringer solution.

\section{Statistical analysis}

All data are presented as mean \pm SEM from the indicated number of experiments "N". Statistical analysis was performed using SigmaStat 3.5 software (SigmaStat software, San Jose, CA, USA). After testing data for normal distribution, the appropriate statistical test was used and the overall $p$ value indicated in the figure legends. Group differences were considered significant when $p$ was $<0.05$, indicated by an asterisk; those at $p$ was $<0.01$ are indicated by double asterisks; those at $p<0.001$ are indicated by triple asterisks. For the Raman spectra, Principal Component Analysis and Linear Discriminant Analysis (PCA-LDA) were performed by means of Origin2018 (OriginLab, Northampton, MA, USA). To test the sensitivity, specificity and accuracy of the classification model to distinguish the EV phenotype by 
the overall biochemical composition, leave-one-out crossvalidation was used.

\section{Results}

\section{EVs bud at the surface of microglia infiltrating the myelin lesion}

Injection of the detergent lysolecithin into the mouse corpus callosum (CC) causes a demyelinating injury within 4 days which spontaneously recovers over time, offering a robust approach to study remyelination [64, 69]. In this model, infiltration and activation of macrophage/microglia were shown to be important for OPC migration and successful differentiation into myelin-forming cells [50, 64].

Ten days after the lesion (dpl), through high-resolution EM analysis, we found cells with the ultrastructural features of microglia (Fig. 1a) along with astrocytes and oligodendrocytes (Fig. 1b) at the lesion site. Importantly, microglia exhibited condensed, electron-dense cytoplasm and nucleoplasm, resembling "dark" microglia, a recently described phenotype of reactive cells [7, 27], characterized by pronounced chromatin remodelling, which is indicative of altered gene expression. Although the narrow conformation of the extracellular space commonly constrains EV visualisation, we observed a dark microglial cell infiltrating the lesion, with several blebs at the cell surface and surrounded by putative membrane vesicles in the pericellular space (Fig. 1c), suggesting intense EV production. This evidence prompted us to investigate the role of EVs in the interplay between reactive microglia and OPCs at lesion sites. Inhibiting endogenous EV production without interfering with the remyelination process is challenging, given that most effective pharmacological or genetic inhibitors of EV biogenesis interfere with sphingolipid metabolism [94]. We, therefore, analysed the action of exogenous EVs produced in vitro by polarised microglia. a

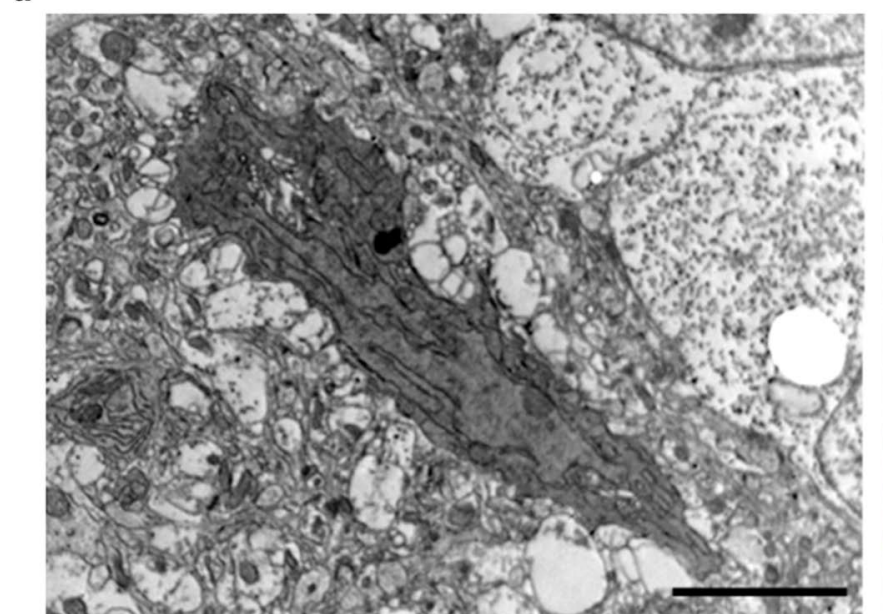

b

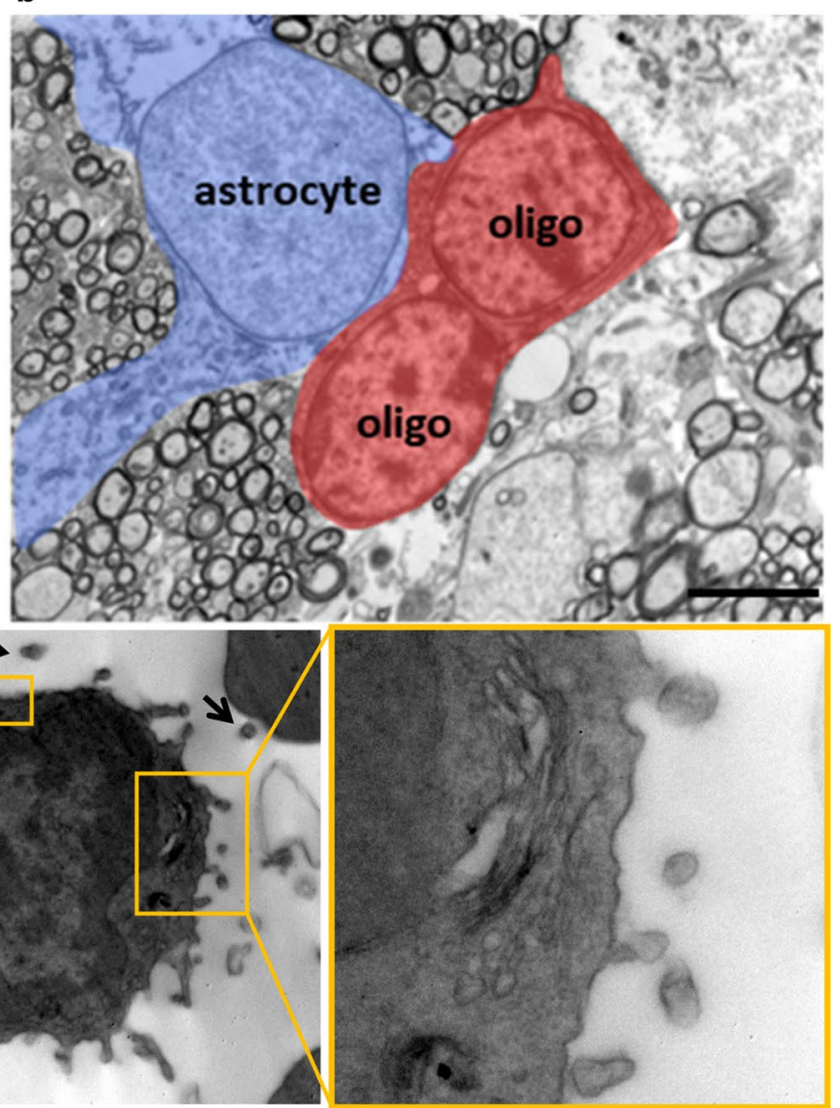

Fig. 1 Microglia infiltrating the myelin lesion release EVs. EM images of the CC showing dark cells resembling microglia (a), oligodendrocytes (b) and astrocytes (b) infiltrating demyelinated lesion at $10 \mathrm{dpl}$. High magnification inserts in $\mathbf{c}$ show examples of EVs budding from the surface of dark microglia (scale bars $2 \mu \mathrm{m}$ ) 


\section{Distinct phenotypic signatures of EVs released by either pro-inflammatory or pro-regenerative microglia}

Primary rat microglia were maintained in the absence of stimuli (NS), under inflammatory (Th1 cytokine cocktail) or pro-regenerative (IL-4) conditions for $48 \mathrm{~h}$. They were also co-cultured with MSCs in transwell system in the presence of inflammatory cytokines (Fig. 2a) to drive proregenerative functions [36, 101]. Gene expression analysis revealed distinct patterns of phenotypic marker expression in the four conditions. Despite common upregulation of pro-regenerative arginase $1(\operatorname{Arg} 1)$ in MSC-treated and IL4-treated microglia, the former ones maintained pro-inflammatory traits (elevated IL-1a, C1q, IL-1 $\beta$ and iNOS) and selectively upregulated a few genes, including Suppressor of cytokine signaling 3 (Socs-3), a gene involved in negative regulation of cytokines and Choline Phosphotransferase 1 (Chpt1), an enzyme involved in phosphatidylcholine synthesis (Fig. 2b).

Non-stimulated (NS) microglia and polarised cells were exposed to ATP for $30 \mathrm{~min}$ to promote $\mathrm{EV}$ release, and EVs were isolated by ultracentrifugation at $100 \mathrm{~kg}$ after preclearing of cell supernatants from cells and debris [34].

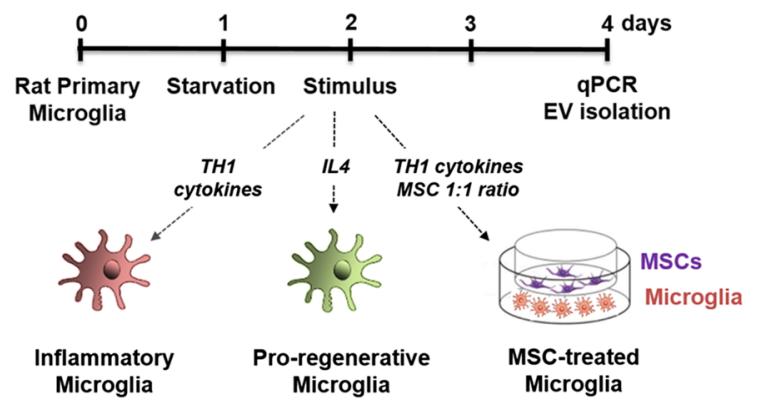

b
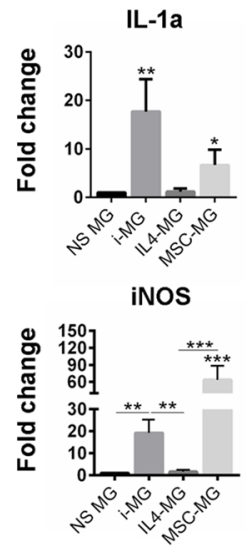
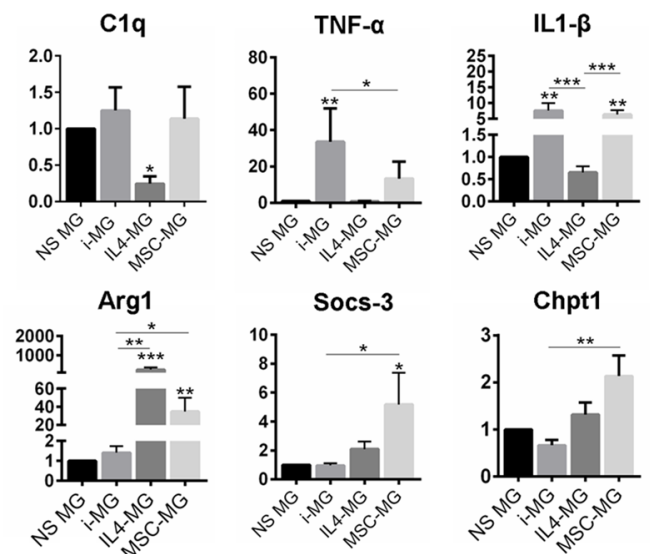

Chpt1

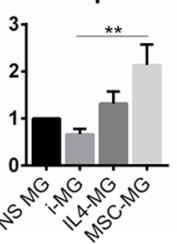

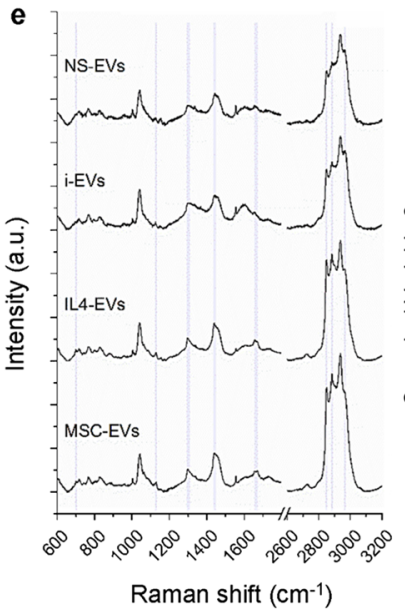

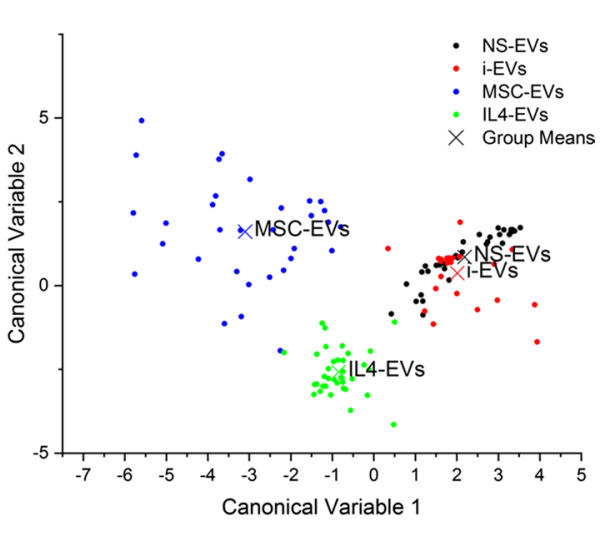

Fig. 2 Production and characterization of EVs released by microglia with different activation states. a Scheme of microglia polarisation in vitro. b Gene expression of inflammatory markers (IL-1a, C1q, TNF- $\alpha$, IL-1 $\beta$ and iNOS), pro-regenerative markers (Arg1 and Socs-3) and the metabolic gene Chpt1 in unstimulated microglia (NS-MG), IL-4-treated microglia (IL-4-MG) or microglia stimulated with inflammatory cytokines in the absence (i-MG) or in the presence of MSCs (MSC-MG) [number of independent experiments $(n)=7-11$ /group; Kruskal-Wallis test with Dunn's multiple comparison $* p<0.05$, ** $p<0.01, * * * p<0.001$. c Representative cryoEM images of the heterogeneous population of microglial EVs in the $100 \mathrm{k} \mathrm{g}$ pellet. d Size profile of EVs pelleted from $1 \times 10^{6}$ microglia, re-suspended in $100 \mu \mathrm{l}$ of $0.1 \mu \mathrm{m}$-filtered PBS and analysed using NTA (top). Histograms show production of EVs from NS or polar- ised microglia during $30 \mathrm{~min}$ stimulation with $1 \mathrm{mM}$ ATP (bottom) ( $n=4$; one way ANOVA $p=0.2898$ with Tukey's multiple comparisons test]. e Mean Raman spectra obtained using $532 \mathrm{~nm}$ laser line from EVs of NS or polarized microglia. All spectra were baseline corrected, aligned and normalised before averaging. f Multivariate statistical analysis performed on the Raman spectra ( $n \geq 30$ per sample). The scatter plot represents the values obtained for the Canonical Variable 1 and Canonical Variable 2 after LDA. In the classification model, spectra from EVs were grouped based on the cell of origin to test RS ability to discriminate the molecular composition of EVs from different microglial phenotypes. The first 10 PC scores calculated by means of PCA were used for the LDA. Each dot represents a single spectrum 
Cryo-electron microscopy (cryo-EM) analysis revealed that most EVs from NS microglia consisted of a single lipid bilayer (Fig. 2c, c", c $c^{\prime \prime \prime} ; 88 \%$ ) and were rounded in shape (Fig. 2c-c"; 66\%), but were very heterogeneous in size, ranging from 15 to $1070 \mathrm{~nm}$ in diameter (mean diameter $81.14 \pm 9.11 \mathrm{~nm}$ ). A subpopulation of EVs contained one or more vesicles in their lumen (10\%; Fig. 2c'), or were coated on their surface (Fig. $2 \mathrm{c}^{\prime \prime} ; 15 \%$ ), likely due to the presence of transmembrane or membrane-associated proteins. According to our previous evidence [34, 95], cryoEM excluded contamination with apoptotic bodies or intracellular organelles derived from damaged cells. Nanosight Tracking Analysis showed no major changes in total EV production from reactive microglia compared to NS cells (Fig. 2d). Conversely, clear differences in the molecular composition of EVs derived from polarised microglia were revealed by Raman spectroscopy (Fig. 2e), a sensitive optical technique already proven to provide information on the chemical content of EVs [39, 40, 51, 85, 88]. Beside the characteristic bands of proteins and nucleic acids (proteins: Amide I $1600-1690 \mathrm{~cm}^{-1}$, Amide III $1200-1300 \mathrm{~cm}^{-1}$; nucleic acids: $720-820 \mathrm{~cm}^{-1}$ ), main differences were identified in the spectral intervals that can be attributed to lipid components (mainly $2700-3200 \mathrm{~cm}^{-1}$ ). In particular, EVs derived from non-stimulated and polarised microglia showed differences in the relative intensities of peaks attributable to cholesterol and cholesterol ester $\left(700 ; 1127 ; 1440 \mathrm{~cm}^{-1}\right)$ and in peaks corresponding to the $\mathrm{CH}_{2}$ deformations (around $1300 \mathrm{~cm}^{-1}$ ) and $\mathrm{CH}, \mathrm{CH}_{2}$, and $\mathrm{CH}_{3}$ bonds (in the spectral range $2600-3200 \mathrm{~cm}^{-1}$ ), which are known to be related to lipids [43]. Despite such similarities, significant differences among IL4-EVs and MSC-EVs spectra were highlighted by linear discrimination analysis, suggesting that the two types of EVs carry distinct cargoes beyond common molecules, thus reflecting the distinct phenotypes of donor cells. The classification model PCA-LDA demonstrated that RS can distinguish the microglia phenotypes of the EV source with an overall accuracy of $91.7 \%$ and an error rate of $18.24 \%$ after cross-validation. The error rate was reduced to $10.16 \%$ when analysing spectra from broken EVs, busted by hypoosmotic shock [34] and re-pelleted to remove soluble components supporting the major contribution of membrane components in the spectral differences between the EV subtypes (Suppl. Figure 1, Online Resource).

\section{Microglia-derived EVs display a dual action on the oligodendroglial response to focal demyelinating lesions}

We first examined the action of EVs produced by inflammatory microglia (i-EVs) and pro-regenerative cells (IL4-EVs and MSC-EVs) on the early response to demyelination (3 $\mathrm{dpl}$ ), during the phase of OPC recruitment [41, 48, 64]. EVs produced by 1.5 million cells $\left(\sim 2-3 \times 10^{8}\right)$ were delivered to focal myelin lesion via pre-filled Alzet mini-pumps for 4 days (at $2 \times 10^{6} \mathrm{EVs} \mathrm{h}^{-1}$ ). EVs were still present in the mini-pumps 3 days after re-suspension, albeit the concentration was reduced by $\sim 30 \%$, as measured by Nanosight Tracking Analysis $\left(\mathrm{EV}\right.$ concentration $=2.80 \times 10^{8} \pm 0.19$ at day $0 ; 2.60 \times 10^{8} \pm 0.13$ at day $1 ; 1.90 \times 10^{8} \pm 0.08$ at day 3$)$. Mice received BrdU twice ( $2 \mathrm{~h}$ apart), at $4 \mathrm{dpl}$, to monitor cell proliferation and the tissue was analysed at the end of the treatment (7 dpl) (Fig. 3a).

Quantification of $\mathrm{BrdU}^{+}$cells showed that all EV types significantly increased cell proliferation in the lesion area compared to saline-injected controls (Fig. 3b). However, we found almost no impact of EVs on the proliferation of Olig2 ${ }^{+}$ oligodendroglial cells (Fig. 3c), indicating that other brain cells proliferate in response to EVs. While not increasing oligodendrocyte proliferation, both IL4-EVs and MSC-EVs increased the density of oligodendroglial cells positive for the transcription factors Sox 10 in the lesion area, suggesting enhanced migration of OPCs to the lesion site (Fig. 3d, e). Staining for myelin protein MBP showed that both IL4-EVs and MSC-EVs promoted the full differentiation of oligodendrocytes, as shown by increased $\mathrm{MBP}^{+}$labelling in the lesioned area (Fig. 3f, g). This maturation effect was accompanied by a decrease in immature NG2 + OPCs $\left(\mathrm{NG}^{+} / \mathrm{Sox} 10^{+}\right.$cells) (Fig. 3e) and an increase in density of oligodendrocytes undergoing differentiation $\left(\mathrm{NG}^{-} \mathrm{Sox} 10^{+}\right.$ cell density: saline, $721.3 \pm 88.64$; i-EVs, $562.0 \pm 40.31$; IL4-EVs, 755.1 \pm 82.13 ; MSC-EVs, $1080 \pm 98.97$; one-way ANOVA main effect of treatment $p=0.0114$ with HolmSidak's multiple comparisons test) upon MSC-EVs delivery. By contrast, the fraction of MBP staining in the lesioned area decreased upon i-EV infusion (Fig. 3f, g) while the tissue exhibited a significant increase in NG2 staining (Fig. 3h, i). Accordingly, the percentage of $\mathrm{NG}^{+} / \mathrm{Sox} 10^{+}$early progenitors increased in comparison to controls (Fig. 3e).

Overall, these data indicate that prolonged delivery of EVs produced by beneficial microglia, especially MSCtreated microglia, promotes the accumulation of oligodendrocytes at the lesion site and enhances remyelination, whereas i-EVs injection inhibits OPC maturation.

To further assess the impact of EVs on OPC differentiation, MSC-EVs and i-EVs $\left(7 \times 10^{7} \mathrm{EVs}\right.$, corresponding to about one third of EVs delivered over 4 days via the minipump) were delivered to LPC-treated mice by a single injection at $7 \mathrm{dpl}$, when OPCs become post-mitotic and progress toward differentiation into myelin-sheath forming oligodendrocytes [64]. The remyelination process was analysed at $10 \mathrm{dpl}$ in both young (2.5-4 months old) and aged (8-12 months old) mice (Fig. 4a), in which remyelination occurs more slowly [44, 82-84]. MRI analysis showed that this time-window is optimal to study remyelination in our experimental setting. Indeed, at 5-6 dpl, before EV 
a

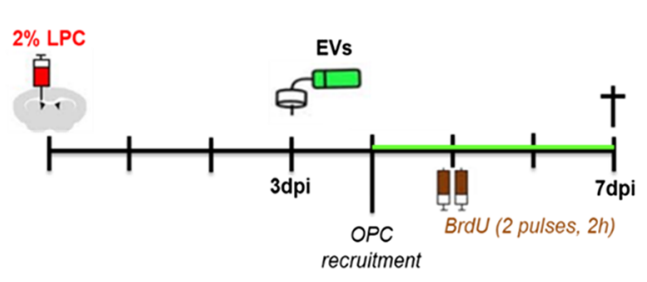

b

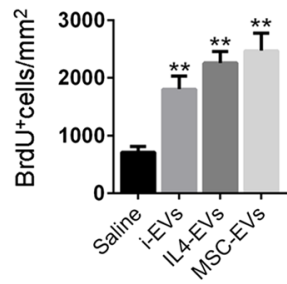

C

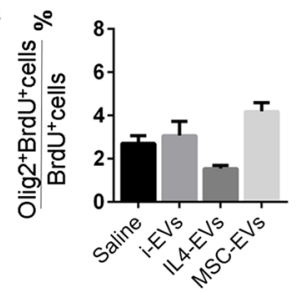

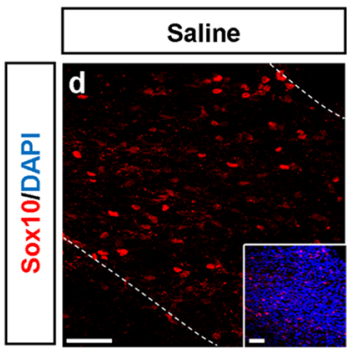
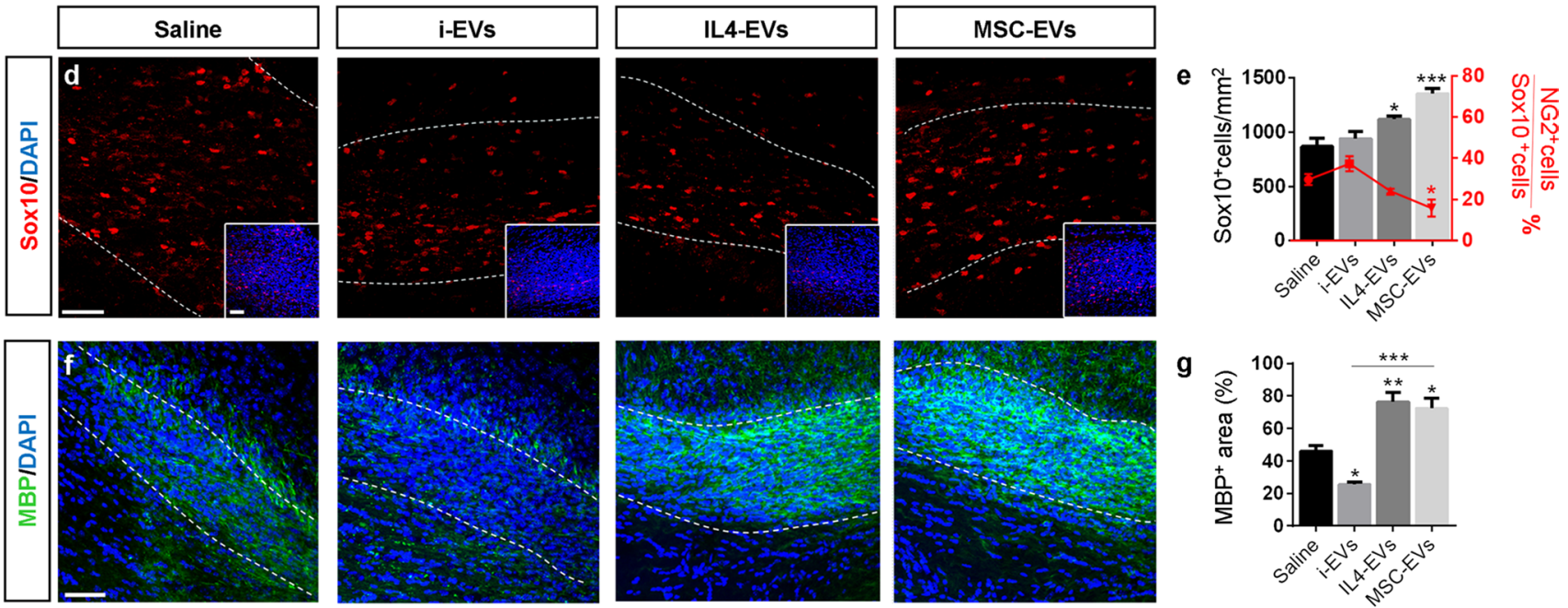

g
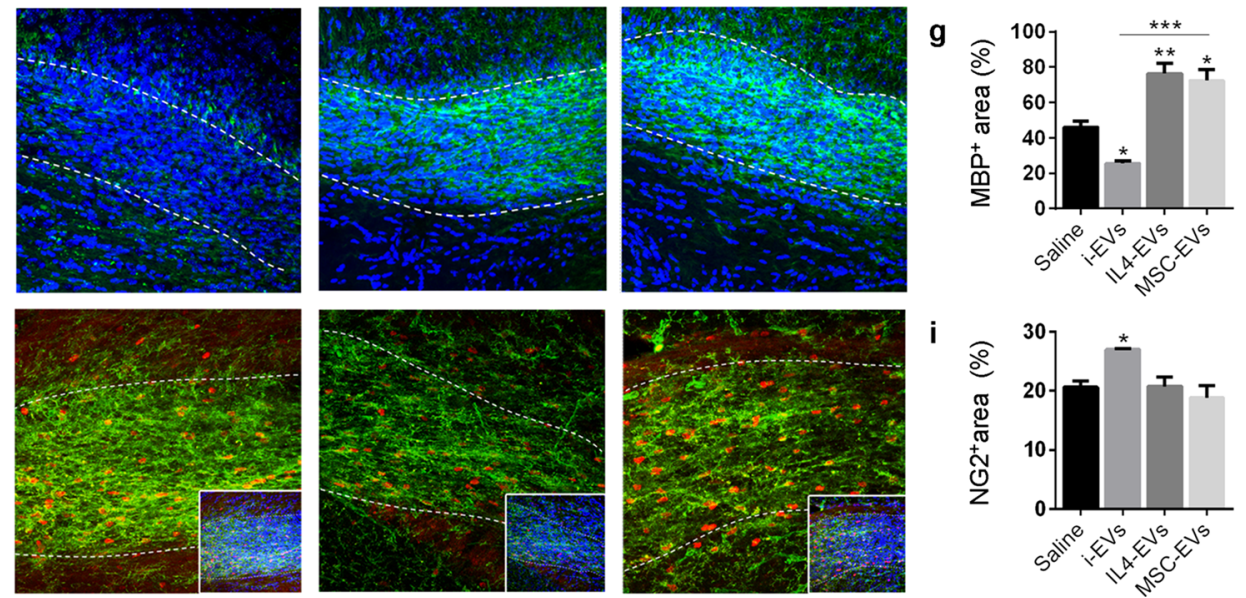

Fig. 3 Action of EVs on early response to EVs. a Experimental design of EV delivery to LPC-treated mice during the phase of OPC recruitment. Histograms show the density of total proliferating cells (b) [number of animals $(N)=3-5 /$ group; one-way ANOVA main effect of treatment $p=0.0021$ with Holm-Sidak's multiple comparisons test vs Saline] and the percentage of $\mathrm{BrdU}^{+}$proliferating OPCs (c) $(N=3-5 /$ group; one way ANOVA $p=0.0165$ with Holm-Sidak's multiple comparisons test vs Saline] in saline, i-EVs- IL4-EVs- or MSC-EVs-injected lesions. d Representative images of Saline, i-EVs, IL4-EVs or MSC-EVs-injected lesions (area delimited by dotted line) immunostained against Sox10 (red) (scale bars of images and insets, $50 \mu \mathrm{m}$ ). Low magnification inserts show Sox10/DAPI double staining to visualise nuclei. e Corresponding density of Sox $10^{+}$ cells (histograms) $(N=3-5 /$ group; one way ANOVA $p=0.0013$ with

treatment, hyper-intense signal on T2-weighted MR images indicated clear myelin lesions in almost the entire CC, whereas at $10 \mathrm{dpl} \mathrm{CC}$ signal returned hypointense almost similar to healthy controls, suggesting fast recovery (Suppl. Figure 2, Online Resource). Remyelination of CC was also observed by diffusion tensor imaging (DTI), showing considerably increased diffusivity perpendicular to white matter fibres at $5 \mathrm{dpl}$, and return to normal values at $10 \mathrm{dpl}$ (Suppl. Figure 2, Online Resource).
Holm-Sidak's multiple comparisons test vs Saline) and percentage of immature $\left(\mathrm{NG}^{+}\right.$Sox $\left.10^{+}\right)$oligodendrocytes (red line) $(N=3-5 /$ group; one way ANOVA $p=0.0074$ with Holm-Sidak's multiple comparisons test vs Saline). f-h Representative images of saline, i-EVs, IL4-EVs or MSC-EVs-injected lesions (area delimited by dotted line) immunostained against MBP (green) and DAPI (blue) (f) or against NG2 (green), Sox10 (red) and DAPI (blue) (h) (scale bars $50 \mu \mathrm{m})$. g, i Histograms show the percentage of the lesioned area immunoreactive for MBP (g) $(N=3-7 /$ group; one way ANOVA $p=0.0001$ with Holm-Sidak's multiple comparisons test vs Saline) or NG2 (i) $(N=3-4 /$ group; one way ANOVA $p=0.0173$ with HolmSidak's multiple comparisons test vs Saline) in saline-injected mice and mice that received different types of EVs

The presence of EVs at the lesion site was verified in mice injected with GFP-labelled EVs produced by GFPexpressing microglia. Twenty minutes after a single injection, GFP-EVs were localised within the lesion evidenced by DAPI staining (Suppl. Figure 3a-d', Online Resource). Immunolabelling for the lineage markers Iba1, S100 $\beta$ or NG2 and 3D reconstruction by Imaris showed GFP-EVs inside microglia/macrophages (Suppl Fig. 3e-e' $\mathrm{e}^{\prime \prime \prime}$, Online Resource), astrocytes (Suppl Fig. 3f-f", Online Resource) 
or $\mathrm{NG}^{+}$cells (Suppl Fig. 3 g-g"', Online Resource). 60 min after injection, GFP-EVs were no longer visible in proximity or away from the injury (not shown). Despite being injected in a single-pulse and in lower amounts compared to the prolonged treatment, both i-EVs and MSC-EVs had a strong impact on oligodendrocytes during the phase of OPC differentiation. In aged mice, i-EVs produced a significant decrease in MBP staining (Fig. 4c, d) with no changes in the fraction of NG2 staining in the lesioned area (Fig. 4e, f) and in the percentage of $\mathrm{NG}^{+}$early progenitors (Fig. 4b). Compared to saline and i-EV injected animals, mice receiving MSC-EVs showed enhanced MBP staining (Fig. 4c, d) and decreased NG2 labelling (Fig. 4e, f), consistent with OPC differentiation into myelin-sheath forming oligodendrocytes. These results were corroborated by the increase in the density of oligodendrocytes positive for $\mathrm{CC} 1$, a myelinating oligodendrocyte marker that stains the cell body [6] (Fig. 4g, $\mathrm{h}$ ), and by the decrease in the percentage of $\mathrm{NG}^{+} / \mathrm{Sox} 10^{+}$ early progenitors at lesion site (Fig. 4b).

In young mice, i-EVs also exerted an inhibitory action on OPC maturation, as indicated by decreased MBP staining at the lesion site (Fig. 5a, b), while the effect of MSCEVs was less evident. Compared to i-EVs, MSC-EVs significantly increased MBP immunoreactivity (Fig. 5a, b), decreased NG2 staining (Fig. 5c, d) and enhanced the density of $\mathrm{CC}^{+}$differentiated oligodendrocytes (Fig. 5e, $\mathrm{f}$ ), but failed to increase remyelination over control levels (Fig. 5a, b, e, f).

Altogether, these data indicate that, while inflammatory microglia inhibit OPC differentiation and remyelination through secretion of i-EVs, MSC-treated microglia release EVs which favor recruitment and transition of NG2 cells into mature myelinating oligodendrocytes at the lesion site.

\section{EVs from different sources induce qualitatively and quantitatively different myelin ultrastuctural features}

For a more detailed definition of myelin ultrastructure, we evaluated the remyelination process by EM analysis at $10 \mathrm{dpl}$ in the $\mathrm{CC}$ of aged lesioned mice that received either i-EVs, or MSC-EVs or saline by a single injection. i-EVs-injected mice displayed a higher $\mathrm{G}$ ratio (Fig. $4 \mathrm{k}-\mathrm{m}$ ) and thinner myelin (Fig. 4l) compared to saline-injected mice, confirming that $\mathrm{i}-\mathrm{EV}$ s hamper remyelination. By contrast, mice injected with MSC-EVs exhibited a higher percentage of myelinated fibres (Fig. 4i, j), lower G ratio (Fig. 4k-m) and higher myelin sheath thickness (Fig. 4l) compared to saline control samples and i-EVs-injected mice, consistent with a promoting action of MSC-EVs on myelin repair.

\section{Strong impact of EVs on OPC migration: the role of sphingosine-1-phosphate}

To investigate the molecular mechanisms underlying harmful and protective action of microglia-derived EVs, we set up primary OPC cultures, a powerful system with easy access for EVs, which allows to dissect their direct effects. All in vitro experiments were performed by exposing OPCs to EVs produced by twice as many microglial cells, in order to keep conditions close to physiology. To further explore how the differential activation state of microglia influences EV action, NS-EVs were included in the analyses.

First, we explored whether EVs have the capacity to influence OPC proliferation and/or migration, two mechanisms involved in oligodendrocyte recruitment into focal myelin lesion. The impact of EVs on proliferation was assessed by exposing cultured OPCs to the different types of EVs for $24 \mathrm{~h}$ in the presence of the proliferative marker EdU. Quantitative analysis of $\mathrm{EdU}^{+}$OPCs showed that i-EVs reduce OPC proliferation, albeit with no statistical significance, while EVs produced by pro-regenerative microglia (either IL4-EVs or MSC-EVs) significantly increased OPC proliferation compared to i-EVs-treated, but not to NS-EVs or untreated (control) OPCs (Fig. 6a, b). In agreement with in vivo observations, these data show very mild effects of EVs on OPC proliferation. By contrast, the impact of EVs on OPC migration was much stronger. Using a classical transwell-based migration assay, we found that all types of EVs significantly enhance the transit of OPCs through the transwell filter, regardless of the activation state of parent microglia (Fig. 6c).

EVs contain components of the sphingolipid pathway, which play a key role in biogenesis and biological action of EVs [94]. Among them, sphingosine-1-phosphate (S1P) is a known chemoattractant agent [49], which may be involved in OPC migration. To test this hypothesis, we pulse-labelled microglia with $\left[{ }^{3} \mathrm{H}\right]$ sphingosine $\left(\left[{ }^{3} \mathrm{H}\right] \mathrm{sph}\right)$ under conditions of metabolic equilibrium and measured the content of $\left[{ }^{3} \mathrm{H}\right]$ sphingolipids in EVs and parental cells. We found detectable levels of $\left[{ }^{3} \mathrm{H}\right] \mathrm{S} 1 \mathrm{P}$ along with more abundant sphingolipids (sphingomyelin, lactosylceramide and monosialodihexosylganglioside (GM3) in EVs produced by NS microglia, but S1P was not enriched in EVs compared to donor cells (Table 3). We next monitored OPC migration in the presence of the pan S1P receptor antagonist S-FTY720-Vinylphosphonate (100 nM) [92] and found that the drug completely inhibited the capacity of EVs to attract OPCs (Fig. 6d).

Collectively, these results indicate that microglial EVs promote OPC migration and highlight a role for vesicular $\mathrm{S} 1 \mathrm{P}$ as attractive guidance cue for OPCs. 
a

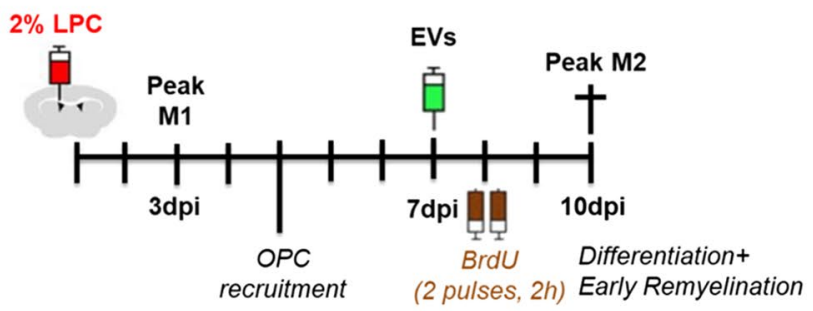

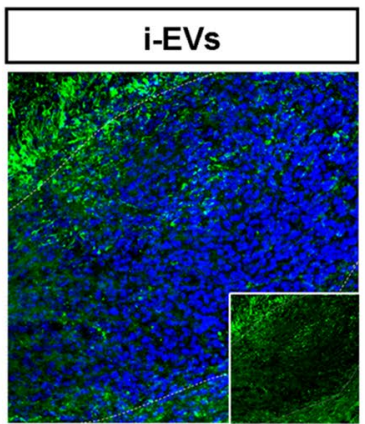
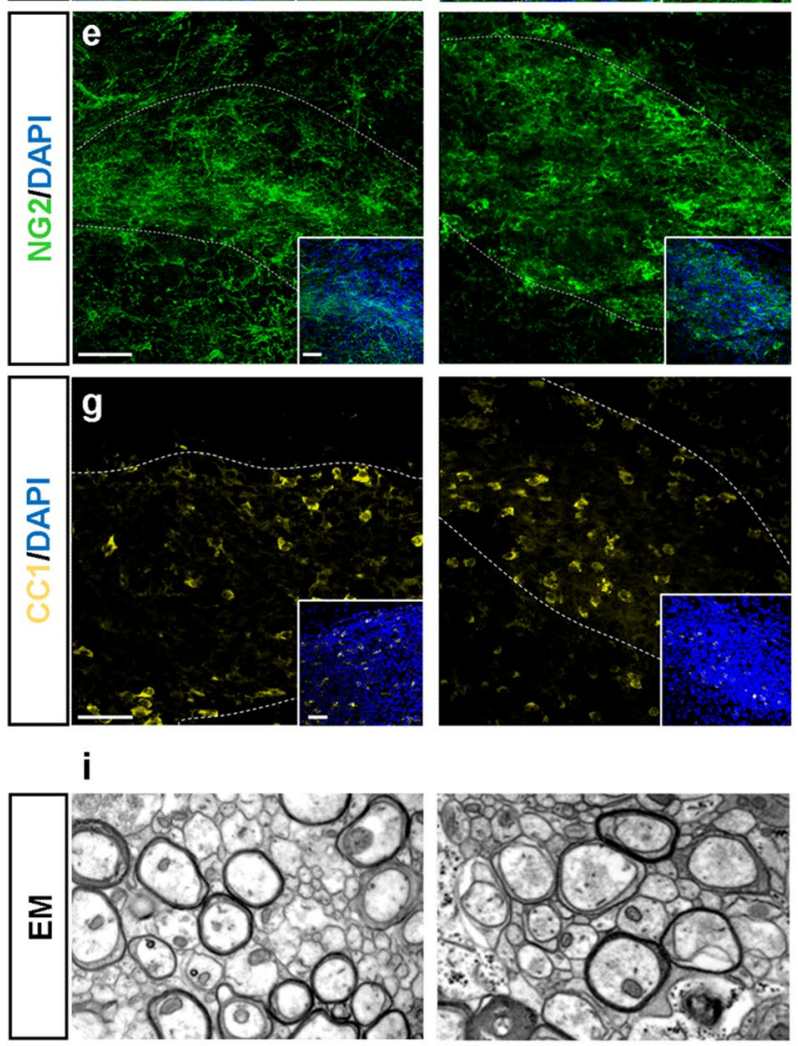

k

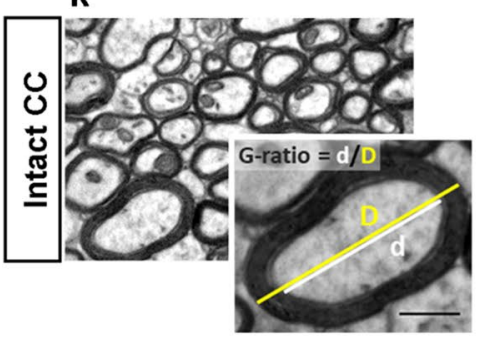

I

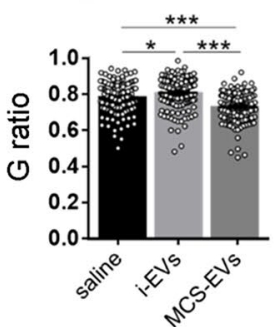

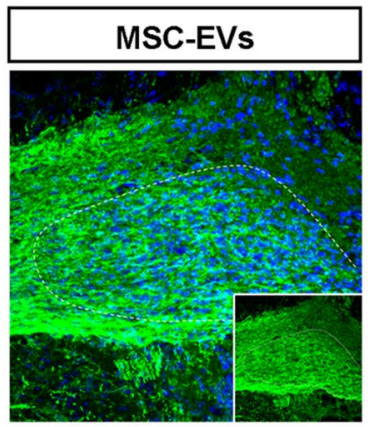
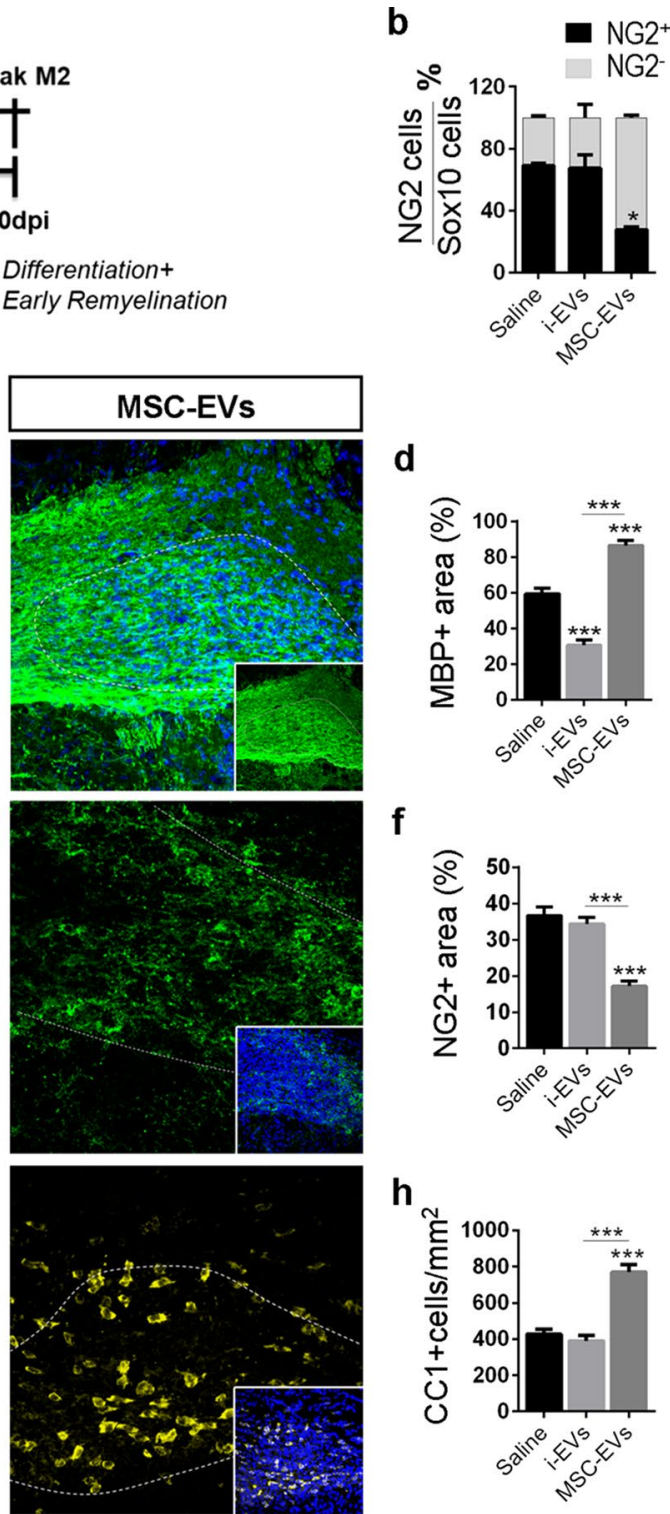

d

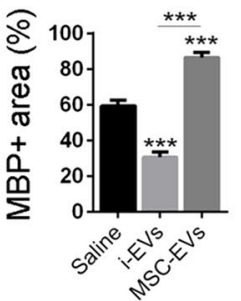

f
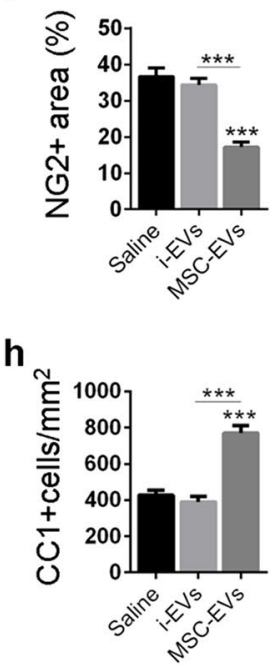

j myelinated

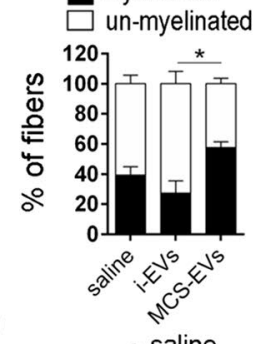

m
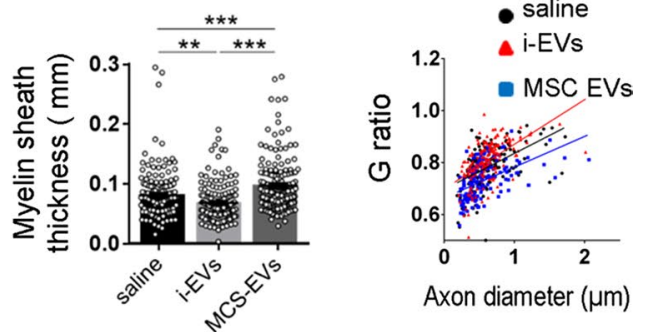
4Fig. 4 Action of EVs on myelin deposition in acute LPC-mediated focal demyelination. a Experimental protocol of EV delivery to LPCtreated mice during the phase of OPC differentiation. b Histograms show percentage of immature $\left(\mathrm{Sox} 10^{+} \mathrm{NG}^{+}\right)$and differentiated cells $\left(\right.$ Sox $\left.10^{+} \mathrm{NG}^{-}\right)$, oligodendrocytes in saline-injected mice and mice that received i-EVs or MSC-EVs $(N=3-4$ /group; Kruskal-Wallis test $p=0.0129$ with Dunn's multiple comparisons test vs Saline). Representative images of saline, i-EVs or MSC-EVs-injected lesions (area delimited by dotted line), immunostained against MBP (green, c), NG2 (green, e), or CC1 (yellow, g) (scale bars $50 \mu \mathrm{m}$ ). Histograms show the percentage of the lesioned area immunoreactive for $\mathrm{MBP}$ (d) $(N=3-4 /$ group; one-way ANOVA $p<0.0001$ with Holm-Sidak's multiple comparisons test), NG2 (f) $(N=4 /$ group; one-way ANOVA $p<0.0001$ with Holm-Sidak's multiple comparisons test) or the density of mature oligodendrocytes (CC1) (h) ( $N=3$ /group; one-way ANOVA $p<0.0001$ with Holm-Sidak's multiple comparisons test. i Representative electron micrographs of CC in saline-injected mice and mice that received i-EVs or MSC-EVs (scale bars of images, $1 \mu \mathrm{m}$; original magnification 25,000). j Histograms show the percentage of unmyelinated/myelinated fibres $(N=3$ / group; one-way ANOVA $p=0.039$ with Dunn's multiple comparison test). k Myelin thickness can be quantified by the $G$-ratio, defined as the ratio between the inner (axonal, $\mathbf{d}$, white) and outer (overlying myelin, d, yellow) diameters of myelinated axons. Scale bar of image, $1.5 \mu \mathrm{m}$. I Histograms show quantifications of the $G$-ratio $(N=3$ /group; Kruskal-Wallis test $p<0.0001$ with Dunn's multiple comparisons test). m Histograms show myelin sheath thickness ( $N=3$ /group; Kruskal-Wallis test $p<0.0001$ with Dunn's multiple comparisons test). Each dot represents an individual value. $\mathbf{n}$ Scatter plots of $G$-ratio against axon diameter. The $G$-ratio of each measured myelinated fibre is indicated by a single circle ( $n=160$ saline, $n=167$ MSC-EVs, $n=169$ i-EVs). Correlation between axon diameter (x1) and $G$-ratio (y1) is expressed by the correlation coefficient $(r)$ of the linear regression curve (saline: $r=0.503$, i-EVs: $r=0.518$; MSCEVs: $r=0.448$ )

\section{In vitro, EVs derived from both inflammatory and pro-regenerative microglia promote OPC differentiation}

To define direct effects of EVs on OPC differentiation, cells were incubated with the different types of EVs for $48 \mathrm{~h}$, fixed and stained for MBP. Unexpectedly, immunofluorescence analysis revealed that $\mathrm{i}-\mathrm{EV}$ s significantly enhanced the fraction of $\mathrm{MBP}^{+}$oligodendrocytes in a similar way to IL4-EVs and MSC-EVs, whereas NS-EVs did not influence OPC maturation (Fig. 6e, f). No significant changes in the fraction of $\mathrm{MBP}^{+}$cells were observed with half or double concentration of IL4-EVs, excluding the possibility that small variations in EV amount could have had a strong impact on OPC maturation (normalized $\mathrm{MBP}^{+}$OPC fraction: $1.53 \pm 0.26$ standard $\mathrm{EV}$ dose; $1.42 \pm 0.22$ half dose; $1.27 \pm 0.36$ double dose). Western blot analysis confirmed that IL4-EVs enhance the expression of MBP along with other markers of mature oligodendrocytes, i.e. 2',3'-cyclic nucleotide-3'-phosphodiesterase (CNPase) and glutathione S-transferase (GST)-pi while downregulating GPR17, a marker of immature oligodendrocytes (Fig. 6g).
Direct action of EVs on myelin deposition was investigated in OPCs co-cultured with DRG neurons, a useful system to study myelination [16]. OPCs were exposed to EVs for 11 days (three treatments, every 3/4 days), fixed and immunostained with axon- and myelin-specific markers, i.e. high-molecular-weight neurofilaments (NF) and MBP, respectively. Quantitative analysis of the linear $\mathrm{MBP}^{+}$segments extending along axons showed that both IL-4-EVs and MSC-EVs favoured myelin deposition (Fig. 6h, i). However, i-EVs also significantly enhanced myelination, albeit at a lower extent compared to pro-regenerative EVs. NS-EVs had no significant effect on myelination (not shown). Of note, MSC-EVs displayed higher capacity to promote myelin deposition compared to EVs released by MSCs (Suppl. Figure 4a, Online Resource).

Globally, these results show that i-EVs exhibit direct prodifferentiating action on cultured OPCs. Thus, the blockade of OPC maturation caused by i-EVs in vivo likely involves other brain cells, which may acquire harmful function in response to i-EVs.

\section{Astrocytes mediate the harmful effects of i-EVs on OPCs}

Recent evidence indicates that microglia can transform astrocytes into A1 harmful cells, which inhibit OPC differentiation [99]. To investigate the possible involvement of astrocytes in the detrimental action of i-EVs, we grew OPCs on top of astrocytes and maintained the co-culture in the presence or absence of i-EVs for $48 \mathrm{~h}$. Immunofluorescence staining for MBP revealed that, in the absence of microglial i-EVs, astrocytes accelerated OPC differentiation, as indicated by higher percentages of MBP/Olig-2 double-positive cells in astrocyte-OPC co-cultures compared to OPCs cultured alone (Fig. 7a, b). However, when exposed to i-EVs, astrocytes caused a strong inhibition of OPC maturation (Fig. 7a, b). Consistent with harmful astrocyte transformation, qPCR analysis showed that, in response to i-EVs, pure cultured astrocytes upregulated the A1 reactive markers serping-1 and Amigo 2 [55] (Fig. 7c), whilst exhibiting unaltered expression for the A2 markers pentraxin-3 (PTX3), CD14 and Tm4sf1 (Fig. 7d). In contrast, the lipid extract of i-EVs potently induced A2 markers (Fig. 7d), and slightly increased serping-1 and Amigo 2 (Fig. 7c), suggesting a shift of astrocytes towards beneficial rather than harmful functions. This indicates that other i-EVs components (proteins and/or RNAs) are mostly responsible for harmful astrocyte transformation. A1 astrocyte conversion likely occurred also in vivo in LPC-treated mice that received i-EVs for 4 days (Fig. 3a). Indeed, GFAP-positive astrocytes, present at myelin lesion, showed decreased PTX3 labelling and increased, albeit non significant, immunoreactivity for the A1 marker C3a compared to saline-injected mice (Fig. 7e, f) 

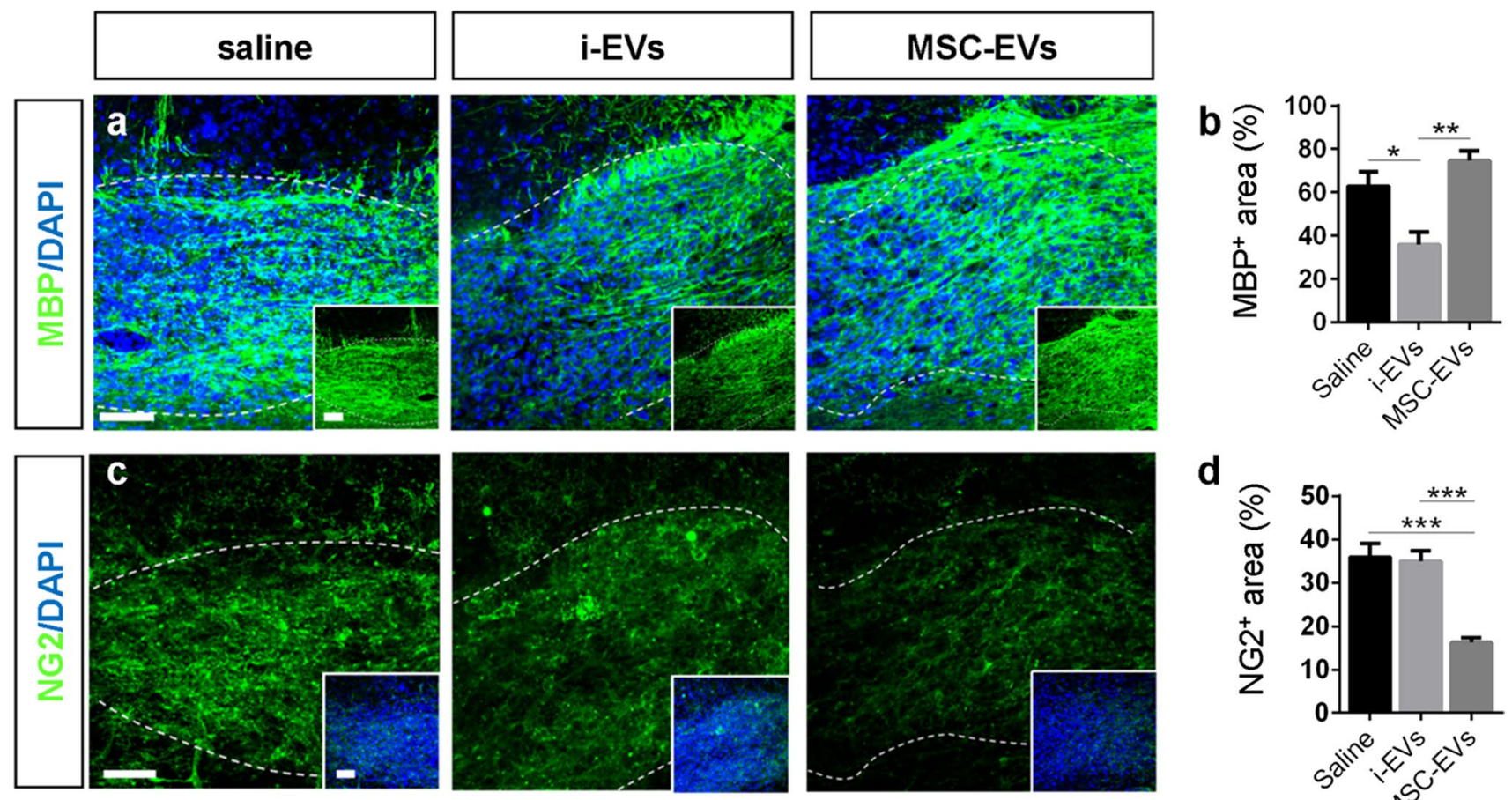

d
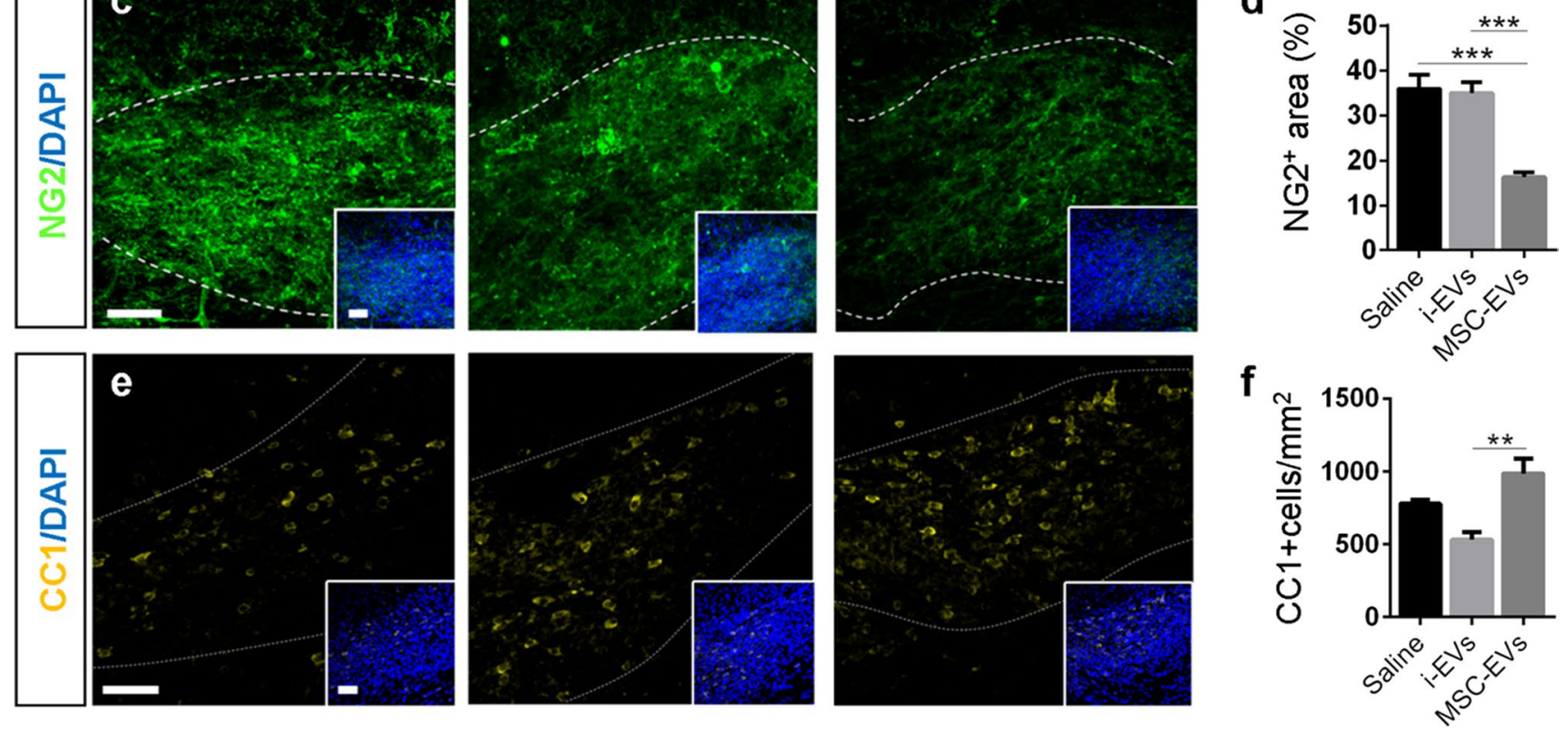

Fig. 5 Action of microglia-derived EVs on OPC differentiation at myelin lesion in young mice. Representative images of saline, i-EVs and MSC-EVs-injected lesions (area delimited by dotted line), immunostained against MBP (green, a), NG2 (green, c), or CC1 (yellow, e) (scale bars $50 \mu \mathrm{m}$ ). Low magnification inserts show double labelling for DAPI. Histograms show the percentage of the lesioned area

No major changes in A1 and A2 markers occurred at protein and mRNA levels in astrocytes exposed to MSC-EVs in vivo and in vitro (Fig. 7c-f).

Three microglial mediators have been shown to be both necessary and sufficient for A1 astrocyte conversion, i.e. IL-1a, C1q and TNF $\alpha$. Of them, TNF $\alpha$ and IL1-a are mostly released by immune cells in EV-associated form $[29,86]$, while both $\mathrm{TNF} \alpha$ and $\mathrm{C} 1 \mathrm{q}$ were previously detected in microglia-derived EVs [17, 25, 98]. To assess the involvement of IL-1a, C1q and TNF $\alpha$ in A1 astrocyte transformation, cytokine profile of i-EVs was assessed with specific ELISA. Detectable amounts of IL-1a, C1q and $\mathrm{TNF} \alpha$ were found in $\mathrm{i}-\mathrm{EV}$ s isolated from $15 \times 10^{6}$ microglial cells (IL1-a $\sim 90$ pg; C1q content $\sim 78$ pg; TNF- $\alpha$ content $\sim 613 \mathrm{pg}$ ), supporting their role in harmful

immunoreactive for MBP (b), NG2 (d) or CC1 (f) at $7 \mathrm{dpl}$ in salineinjected mice and mice injected with i-EVs or MSC-EVs [number of animals $(N)=3-5 /$ group, one-way ANOVA with Holm-Sidak's multiple comparison: MBP, $p=0.0012$; NG2, $p<0.0001$; CC1, $p=0.0090)$

astrocyte transformation. Among the three inflammatory mediators, only TNF- $\alpha$ was suppressed in parental microglia by MSC conditioning at both protein and mRNA levels, as determined by ELISA (Fig. 7g) and qPCR (Fig. 2b). We, therefore, measured the TNF $\alpha$ content in i-EVs and MSC-EVs. i-EVs displayed about threefold higher levels of TNF- $\alpha$ compared to MSC-EVs (i-EVs TNF- $\alpha$ content $\sim 613 \mathrm{pg}$; MSC-EVs TNF- $\alpha$ content $\sim 168 \mathrm{pg}$ ), suggesting that the capacity of MSC-EVs to promote myelin repair may occur, at least in part, via TNF- $\alpha$ suppression. To assess TNF- $\alpha$ involvement in astrocyte activation, we pre-treated i-EVs with Etanercept (ETN, $200 \mathrm{ng} \mathrm{ml}^{-1}$ ), a TNF- $\alpha$ inhibitor, before co-exposing astrocytes to i-EVs and ETN. qPCR analysis showed that TNF- $\alpha$ inactivation prevented serping-1 upregulation and induced PTX3 
expression in vitro, suggesting a shift of cultured astrocytes towards beneficial function (Fig. 7c, d). Immunolabelling for $\mathrm{C} 3$ in vivo revealed a significant decrease in the density of astrocytes positive for the A1 marker at lesions co-injected with i-EVs and ETN compared to i-EVs alone. However, PTX3 staining showed that the inhibitor caused by itself a decrease in the density of PTX3 positive astrocytes, being not able to prevent the decrease in PTX3 ${ }^{+}$ astrocytes induced by i-EVs (Fig. 7e, f). Taken together, these data indicate that TNF- $\alpha$ is essential for the astrocyte response, despite its inactivation is not sufficient to prevent harmful astrocyte transformation in vivo.

\section{EVs binds to the OPC cell surface}

While mechanisms underlying astrocyte reaction to microglial EVs have been previously studied [25, 95], how EVs influence OPC activity is completely unknown. We explored whether EVs physically interact with OPCs. We placed single EV in contact with cultured OPCs using optical tweezers and monitored EV-OPC dynamics using time lapse microscopy (Fig. 8a). We observed that about $70 \%$ of NS-EVs adhered to the OPC surface (17 out of 24), suggesting that, in principle, most EVs could influence OPC function.

\section{The pro-differentiation activity of EVs depends on the lipid components of EVs}

To investigate whether direct effects of EVs were mediated by factor(s) present at the surface or in the lumen of EVs, IL-4-EVs or MSC-EVs were busted by hypo-osmotic shock [34] and membrane fragments pelleted to remove soluble components were added to cultured OPCs. Broken EVs, depleted of their content, retained the capability to promote OPC maturation (Fig. 8d), revealing that surface component(s) of EVs were mainly driving this process. To distinguish between protein and lipid component(s), we exposed OPCs to the lipid fraction of IL4-EVs (Fig. 8e) or i-EVs (not shown). EV lipids enhanced OPC maturation even more efficiently than intact EVs (Fig. 8e), indicating that the pro-differentiating activity of EVs is mostly attributable to their lipid components. Finally, given that previous studies have shown that S1P and its analog FTY720-p promote OPC differentiation and myelin deposition [23, 65], we asked whether vesicular S1P may be implicated in the prodifferentiating activity of the EVs. We treated OPCs with the S1P receptor antagonist S-FTY720-Vinylphosphonate and found no changes in EV-induced OPC differentiation to $\mathrm{MBP}^{+}$oligodendrocytes (Fig. 8f), thus ruling out a role of vesicular S1P in OPC maturation.

\section{Discussion}

Our study reveals a previously unrecognised role of EVs produced by microglia in the control of remyelination, the spontaneous process by which OPCs differentiate into myelin-forming cells, restore myelin sheaths to protect axons from degeneration and allow fast signal transmission [24]. Specifically, we show that exogenous EVs from differentially conditioned microglia exert opposite effects on remyelination, with either pro-regenerative or detrimental actions, depending on the type of conditioning stimulus. Importantly, our study also unveils an unprecedented role for astrocytes in mediating the detrimental effects of EVs from pro-inflammatory microglia. Finally, it gives a first identification of the distinct molecular components of EVs involved in astrocyte detrimental transition as well as in promotion of OPC migration and/ or differentiation, thus unveiling new targets for modulation of myelin repair.

\section{EVs mediate microglial action on oligodendrocytes}

Previous evidence had shown that microglial phenotype strongly influences remyelination $[30,72]$ and that a predominant pro-regenerative response of microglia is required for efficient myelin repair after damage [64]. However, the mode(s) of action of microglia in fostering or inhibiting myelin repair was largely unclear. In the present study, by utilising a combined approach (immunohistochemistry and EM), we unequivocally show that EVs released by pro-regenerative microglia promote OPC recruitment and differentiation at LPC-induced myelin lesions, while i-EVs, derived from inflammatory microglia, block remyelination. Our study advances current knowledge by showing that (1) dark, reactive microglia are recruited at myelin lesion and release EVs, as indicated by EM, and (2) EVs are sufficient to mediate either detrimental or beneficial function on myelin forming cells, reflecting the phenotype of donor microglia, upon exogenous administration to the lesion site. EVs remain intact for a short time at the injection site, likely as a consequence of lysosomal degradation [15] or cell fusion and cargo dilution [75], but cause a prolonged modulation of oligodendrocytes surrounding the lesion. This is in line with a recent study showing that microglial EVs engineered to deliver an anti-inflammatory cytokine IL-4 induce a longlasting immune modulation in the EAE model of multiple sclerosis [15]. However, whether EVs with opposite action on OPCs may be generated endogenously by microglia and may be relevant for the course of de- and re-myelination in animal models of MS still remains unclear. Selective 

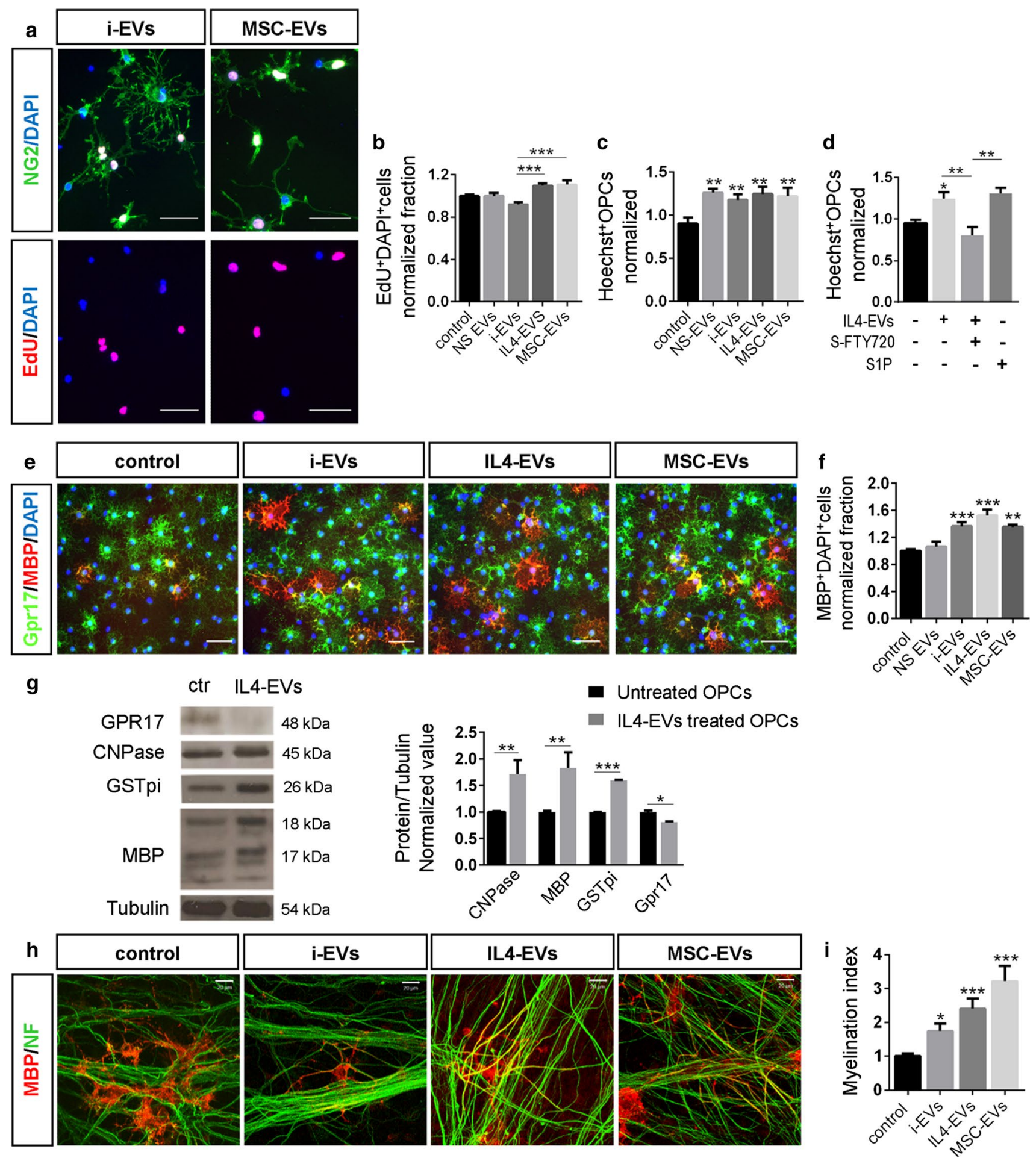

tools to manipulate endogenous EV production are needed to overcome this limitation of our study and to analyse the role of microglial EVs in a more physiological setting.

\section{A1 astrocytes mediate detrimental action of i-EVs on OPCs}

The major finding of this paper is the demonstration that astrocytes mediate the detrimental action of inflammatory microglia-derived EVs on OPCs. While i-EVs by themselves favor differentiation of OPCs in monoculture, they 
4Fig. 6 EV impact on OPC migration, differentiation and myelination. a, b Fluorescence images of cultured OPCs incubated with EdU (red), fixed and stained for NG2 (green) and DAPI (blue) after $24 \mathrm{~h}$ exposure to i-EVs or MSC-EVs (scale bars $50 \mu \mathrm{m}$ ). The histograms in $\mathbf{b}$ show the percentage of $\mathrm{EdU}^{+} \mathrm{OPCs}$ in cultures exposed or not to different EV types. Data have been normalized to control [number of experiments $(n)=5-8 /$ group; Kruskal-Wallis test $p<0.0001$ with Dunn's multiple comparisons test]. c Histograms show the percentage of OPCs migrated through the filter of the Boyden chamber in control conditions and following addition of different types of EVs. Data have been normalized to control $(n=3$; one-way ANOVA $p=0.0054$ with Holm-Sidak's multiple comparisons test vs control). d Percentage of migrated OPCs in response to S1P or IL-4-EVs in the presence or in the absence of the S1P receptor antagonist S-FTY720Vinylphosphonate $(n=3$; Kruskal-Wallis test $p<0.0001$ with Dunn's multiple comparisons test). e Representative images of OPCs maintained in control conditions or exposed to different types of EVs for 2 days, fixed and stained for MBP (red), GPR17 (green) and DAPI (blue) (scale bars $50 \mu \mathrm{m}$ ). f Corresponding quantification of $\mathrm{MBP}^{+}$OPCs. Data have been normalized to control ( $n=5-8 /$ group, Kruskal-Wallis test $p<0.0001$ with Dunn's multiple comparisons test vs control). g Western blot of control OPCs and IL4-EVs-treated OPCs for the indicated markers of OPC differentiation. Tubulin has been used as loading control. Relative quantification of the band density is shown on the right ( $n=3$; CNPase: unpaired $t$ test $p=0.0033$; MBP: unpaired $t$ test $p=0.0018$; GST-pi: unpaired $t$ test $p<0.0001$; GPR17: unpaired $t$ test $p=0.0224)$. h Representative images of OPCDRG co-cultures maintained in control conditions or exposed to i-EVs, IL4-EVs or MSC-EVs for 11 days, fixed and stained for MBP (red) and neurofilament (NF, green) (scale bars $20 \mu \mathrm{m}$ ). e Myelination index (MBP staining/NF staining) under different experimental conditions $(n=3$; Kruskal-Wallis test $p<0.0001$ with Dunn's multiple comparisons test vs control)

Table 3 Sphingolipid content in EVs and parental microglia

\begin{tabular}{lrrl}
\hline Sphingolipid species & \% in cells & $\%$ in EVs & $\begin{array}{l}\text { EVs/ } \\
\text { cell fold } \\
\text { increase }\end{array}$ \\
\hline$\left[{ }^{3} \mathrm{H}\right]$ Sphingomyelin & $27.55 \pm 2.4$ & $33.88 \pm 3.4$ & 1.2 \\
{$\left[{ }^{3} \mathrm{H}\right]$ Ceramide } & $4.04 \pm 0.4$ & $4.60 \pm 0.4$ & 1.1 \\
{$\left[{ }^{3} \mathrm{H}\right]$ Glucosylceramide } & $5.97 \pm 0.5$ & $4.41 \pm 0.4$ & 0.7 \\
{$\left[{ }^{3} \mathrm{H}\right]$ Lactosylceramide } & $19.26 \pm 1.8$ & $11.41 \pm 1.4$ & 0.6 \\
{$\left[{ }^{3} \mathrm{H}\right]$ Sphingosine } & $3.78 \pm 0.4$ & $4.31 \pm 0.5$ & 1.1 \\
{$\left[{ }^{3} \mathrm{H}\right]$ GM3 } & $10.4 \pm 0.6$ & $8.94 \pm 1.0$ & 0.8 \\
{$\left[{ }^{3} \mathrm{H}\right]$ Sphingosine-1-phosphate } & $1.17 \pm 0.14$ & $0.95 \pm 0.15$ & 0.8 \\
\hline
\end{tabular}

cause a clear block of OPC maturation when OPCs are cocultured with astrocytes, thereby mimicking the inhibitory action of i-EVs at myelin lesion in the in vivo LPCdemyelination model. Importantly, inhibition of OPC maturation in the OPC/astrocytic co-cultures is likely paralleled by conversion of astrocytes into A1 harmful cells. Indeed, our previous work revealed that cultured astrocytes become hypertrophic and upregulate inflammatory markers when exposed to i-EVs [95]. By analysing the expression and immunoreactivity of a few specific A1 and A2 markers in this study, we suggest that astrocytes acquire a harmful A1 phenotype both in vitro and in the LPC-mouse model of myelin lesion. A1 astrocytes were recently proven to slow OPC differentiation and to damage differentiated oligodendrocytes [20,99] and have been observed in demyelinating plaques of MS patients [55]. By showing that microglial i-EVs cause A1 astrocyte transformation, we add an important piece of information towards the understanding of the mechanism possibly responsible for remyelination failure in MS. We hypothesise that demyelinating lesions may fail to remyelinate because $\mathrm{EV}$ s produced by chronically activated microglia block OPC differentiation by inducing harmful astrocyte conversion, thus nullifying the direct pro-myelinating action of EVs. In line with our study, previous works highlighted the detrimental effect of chronically activated astrocytes on myelin repair, by showing that depletion of reactive astrocytes at the chronic phase of EAE improves EAE clinical outcome [62] and that remyelination by transplanted OPCs is less extensive in areas of demyelination that contain astrocytes than in astrocyte-free areas [8]. A few proteins released by astrocytes, including CXCL10 [67] and endothelin-1 [42], inhibit OPC maturation and may be involved in remyelination failure. By contrast, no molecules with the capacity to damage OPCs/oligodendrocytes have been identified so far in the secretome of inflammatory microglia, which rather contains protective agents for myelin-forming cells [24, 63].

\section{EV signals driving astrocytes towards oligotoxic cells}

Although our analysis indicates that EVs carry the glycolipid lactosylceramide (LacCer) and S1P, known inducers of pro-inflammatory astroglial activation in EAE [19, $62,81]$, the lipid extract of i-EVs does not drive harmful transformation in cultured astrocytes, implicating the protein and/or RNA cargoes of i-EVs in the acquisition of detrimental astroglial function. Previous studies reported that activated microglia release three mediators that are necessary and sufficient for astrocyte conversion to harmful cells, i.e. TNF- $\alpha$, IL-1a and C1q [55]. Here we show that (1) i-EVs carry the three inducers of A1 astrocytes, (2) TNF- $\alpha$ is decreased in MSC-EVs, which do not cause harmful astrocyte transformation, and (3) TNF- $\alpha$ inactivation partially inhibits the proinflammatory astrocyte response. Besides identifying TNF- $\alpha$ as a target of MSC action in microglia, this evidence implicates vesicular TNF- $\alpha$ in the astrocyte shift towards oligotoxic cells. However, other molecules may be sorted in MSCEVs, such as TGF- $\beta$ or FGF [2], which may counteract the action of the inflammatory cargoes, preventing A1 astrocyte activation. 

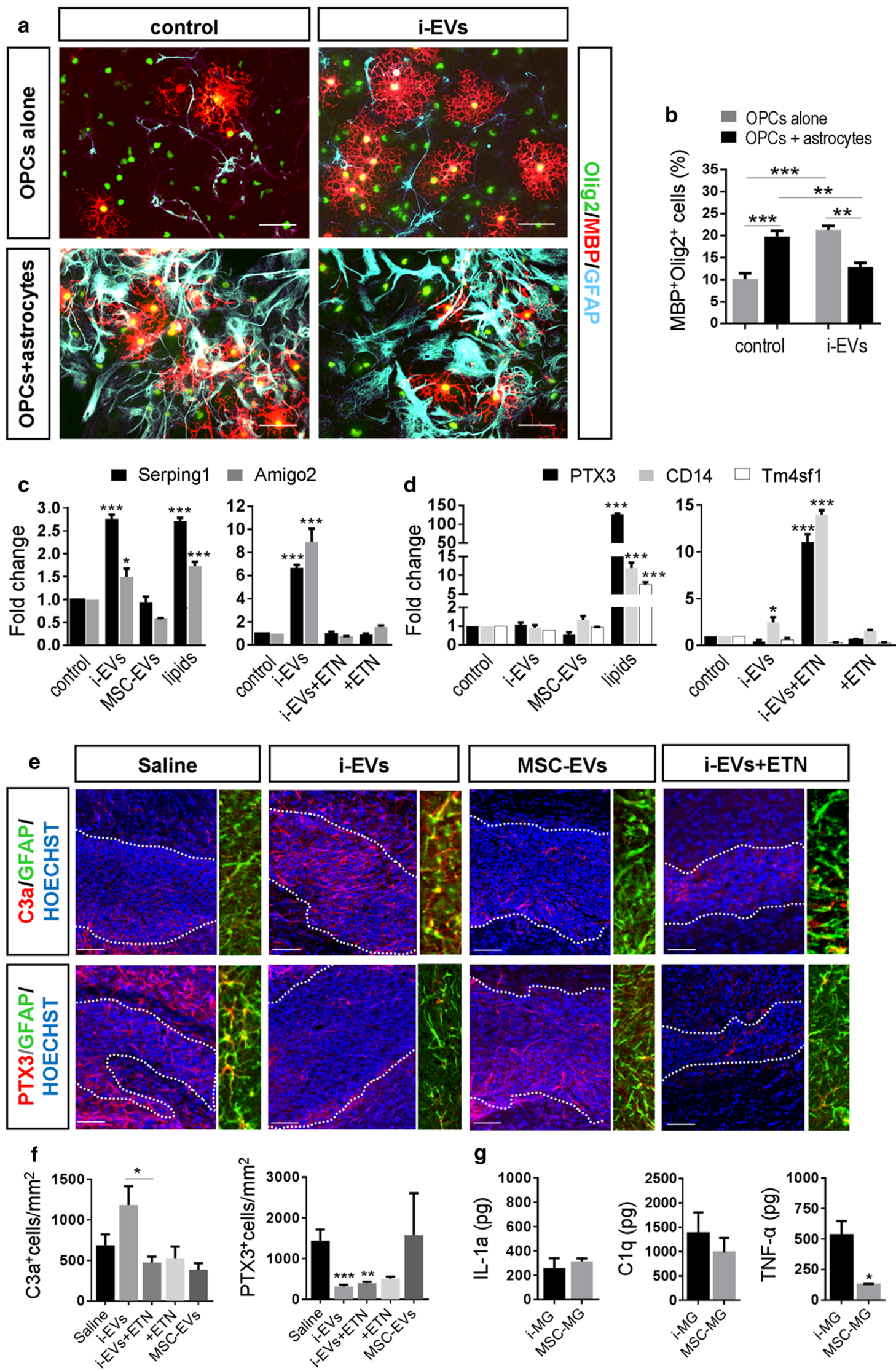
4Fig.7 Astrocytes transform the pro-differentiating action of i-EVs to inhibitory activity. a Representative images of OPCs cultured alone or with astrocytes in the presence or in the absence of i-EVs immunostained for MBP (red), Olig2 (green) and GFAP (cyan) (scale bars $20 \mu \mathrm{m})$. b Corresponding quantifications of $\mathrm{MBP}^{+} \mathrm{OPCs} \quad(n=2$; one-way ANOVA $p<0.0001$ with Tukey's multiple comparisons test). Representative qPCR analysis of A1 (c) and A2 (d) marker expression in unstimulated astrocytes (control), astrocytes exposed to i-EVs, the lipid fraction of i-EVs (lipids), MSC-EVs (left panels). Right panels show A1 and A2 marker expression in astrocytes exposed to $\mathrm{i}-\mathrm{EV} \mathrm{s}$ in the presence/absence of the TNF-a inhibitor etanercept $(\mathrm{ETN})$.Three replicates/condition have been normalized to control (one-way ANOVA $p<0.0001$ with Holm-Sidak's multiple comparisons test). e Representative images of saline, i-EVs, or MSC-EVs-injected lesions, immunostained against C3 or PTX3 (red) and Hoechst (blue) (scale bars $50 \mu \mathrm{m}$ ). High magnification inserts show astrocytes double stained for GFAP (green) and C3 or PTX3 (red). f Density of C3- and PTX3-positive astrocytes at saline, i-EVs-, i-EVs +ETN, or MSC-EVs-injected lesions (C3, number of sections $=5-10$ /group; Kruskal-Wallis test $p=0.0298$ with Dunn's multiple comparisons test among Saline, i-EVs and i-EVs +ETN); (PTX3, number of sections $=5-10 /$ group; Kruskal-Wallis test $p<0.0001$ with Dunn's multiple comparisons test). g ELISA quantification of IL-1a, C1q, and TNF- $\alpha$ in $1 \times 10^{6}$ inflammatory microglia (i-MG) and in MSC-treated microglia (MSC-MG) $(n=3$; IL-1a, Mann-Whitney $t$ test $p=0.9000 ; \mathrm{C} 1 \mathrm{q}$, unpaired t test $p=0.5619$; TNF- $\alpha$, Mann-Whitney $t$ test $p=0.0286$ )

Elevated production of TNF- $\alpha$ was previously reported in the serum, cerebrospinal fluid and brain plaques of MS patients [59], correlating with disease severity or exacerbation $[58,93]$. In addition, it was recently shown that TNF- $\alpha$ is mostly produced by microglia and macrophages in acute and progressive EAE, enhancing clinical disability [91]. Whether the content of TNF- $\alpha$ is elevated in microglial EVs of MS patients, especially those with progressive MS, is an important question for future studies that may lead to the identification of novel disease biomarkers. In devising possible therapeutic strategies to promote myelin repair, it should be noted, however, that TNF- $\alpha$ has been attributed both detrimental and protective roles in MS. Specifically, the membrane bound form of the cytokine was shown to sustain reparative processes via activation of type 2 TNF- $\alpha$ receptor (TNFR2) in oligodendrocytes [13], preventing the clinical use of TNF- $\alpha$ blockers in MS [47].

\section{Key role of lipids in the pro-myelinating action of EVs}

Another key observation of our study is that the lipid content of all types of microglial EVs has a direct impact on OPCs, directing both their recruitment and differentiation.

Through measurement of sphingolipid content and the use of a potent S1P receptor antagonist S-ene-FTY720 Vinylphosphonate, we show that S1P is secreted by microglia in association with EVs and acts as an attractive guidance cue for OPCs. Although the recent use of the S1P analog fingolimod/FTY720, which activates S1P receptors, to treat MS has fueled research on the direct effects of S1P on oligodendrocytes [38], to our knowledge, our data provide the first demonstration of a central role for EVs-associated S1P in OPC migration, the first key step in myelin repair. Other chemotactic molecules, such as Wnt3a [45] and the endocannabinoid anandamide [34] are packaged in microglial EVs, revealing the important role of EVs as vehicles of chemotactic signals [52]. Through EV fractionation, we also show that lipids of all EV types drive OPC maturation, making lipid research a hotspot in the field of myelin repair. We rule out the involvement of S1P in the pro-differentiating action of EVs, since the capacity of EVs to accelerate OPC maturation was unchanged under pharmacological blockade of S1P receptors. We anticipate that identification of lipids responsible for the pro-differentiating action of microglial EVs may help to design new therapeutics to foster myelin repair.

Previous studies implicated the miRNA cargo of EVs in myelin repair, rather than the lipid content. Pro-myelinating exosomes derived by peripheral immune cells were previously identified in rat serum $[76,77]$. Their pro-differentiating activity was partially ascribed to the delivery of miR219 to OPCs, a microRNA that regulates multiple genes in the differentiation pathway [76]. Although miR-219 is present in EVs derived from microglia and is upregulated in i-EVs [75], our biochemical experiments indicate that the lipid fraction of EVs, which is free of nucleic acids, retains the ability to drive OPC maturation, arguing against a major involvement of miRNAs in the direct pro-differentiating action of microglial EVs. Nevertheless, the RNA and/or protein cargoes of EVs may indirectly regulate OPC maturation by influencing the microenvironment around the lesion.

\section{The unique pro-myelinating activity of EVs derived from microglia co-cultured with MSC}

We show that MSC-EVs have a unique capacity to stimulate the endogenous reparative response of OPCs at myelin lesion. The pro-myelinating action of MSC-EVs exceeds that of IL4-EVs both in vitro and in vivo. This suggests that well-known effects of MSCs in EAE rely, at least in part, on the paracrine effect of MSCs on microglia phenotype and microglia communication with OPCs and astrocytes during remyelination. Further work remains to be done to define the minimal components of MSC-EVs required to drive efficient remyelination. Selected luminal components of MSC-EVs (proteins and/or RNAs) in combination with pro-differentiating lipid(s) may be assembled in EV mimetics of low complexity and with low chance of off-target effects, which 

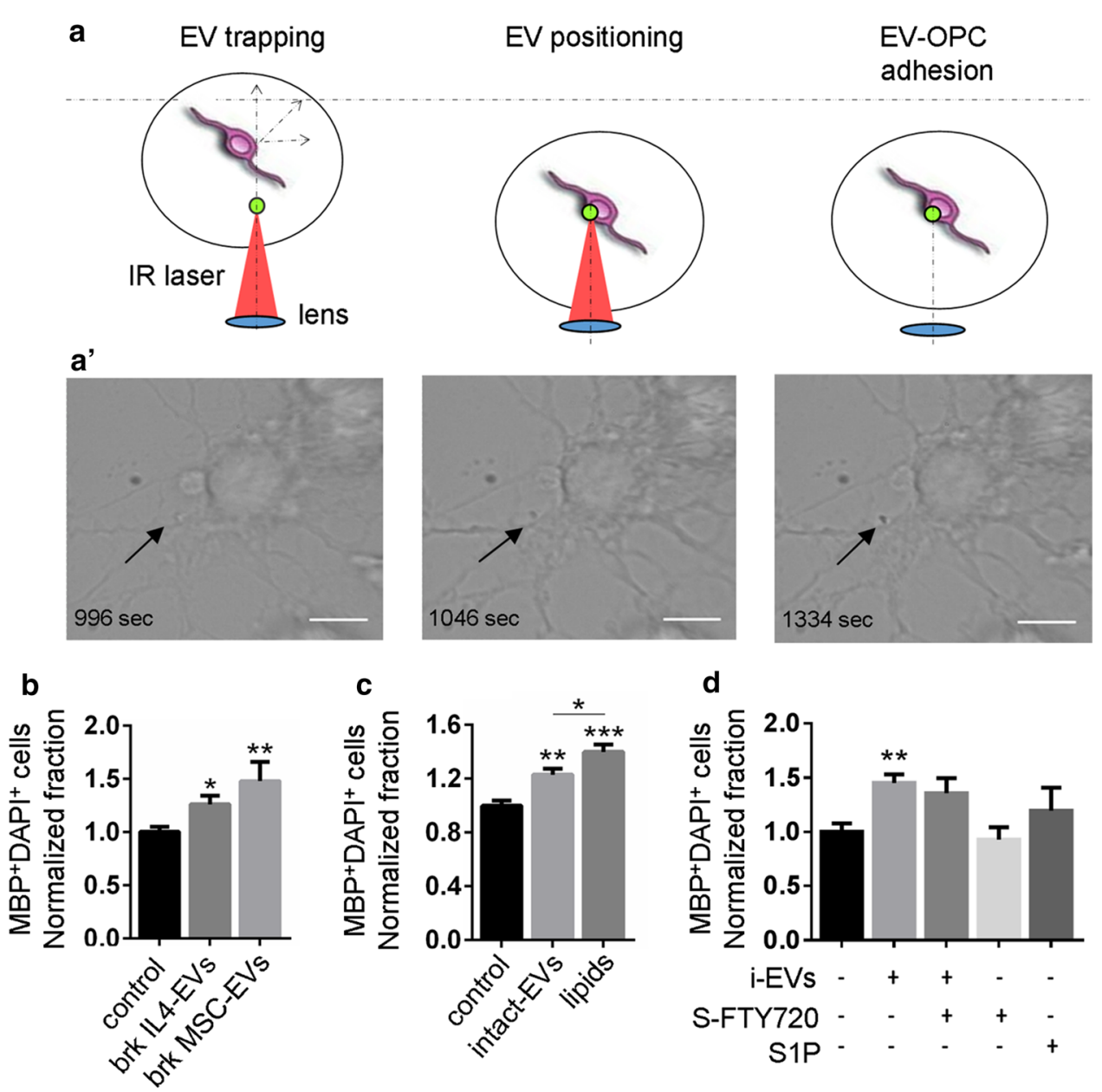

Fig. 8 EVs efficiently interact with OPCs. a Schematic representation of EV delivery to OPCs by optical tweezers. EVs are first trapped above the OPCs by the IR laser tweezers (left), then the stage is moved in plane $(X Y)$ and the objective/trap is moved axially $(Z)$ to set the EVs in contact with the OPCs (middle). The trapping laser is switched off to check whether EV adheres to the neuron membrane (right). a' Sequence of phase-contrast images showing one example of EV driven to an OPC following the procedure described in a (scale bar $10 \mu \mathrm{m}$ ). b, c OPCs were maintained in control condition or exposed to intact EVs, broken EVs or the lipid extract of EVs for 2 days, fixed and stained for MBP. Histograms show the percentage of mature $\mathrm{MBP}^{+}$oligodendrocytes in cultures exposed to broken

may be ideal tools to develop novel therapeutic approaches in chronic MS.

Acknowledgements We are grateful to Drs. Roberto Furlan (San Raffaele Scientific Institute, Milan, Italy) and Bobbi Fleiss (RMIT University, MelburneMelbourne, Australia) for helpful discussion. We thank Drs. Laura Pergoli (University of Milan, Milan, Italy) for help with NTA of EVs, Paolo Swuec (University of Milan, Milan, Italy) for cryoelectron microscopy analysis of EVs and Stefano Raffaele (University of Milan, Milan, Italy) for help with some IHC experiments. We are grateful to Dr. Francesca Garello and Prof. Enzo Terreno (University of Turin, Italy) for assistance with MRI analysis of myelin lesions. The project has been supported by GMSI 2015 to $\mathrm{CV}, \mathrm{AB}, \mathrm{MF}$ and $\mathrm{AU}$ and FISM/2018/R/22 to CV. MG was supported by a FISM Fellowship
EVs derived from IL-4 microglia (Brk IL4-EVs) or MSC-treated cells (Brk MSC-EVs) $(\mathbf{b} ; n=3$; Kruskal-Wallis test $p=0.0049$ with Dunn's multiple comparisons test versus control), intact EVs or native lipids (lipids) extracted from IL4-EVs (c; one-way ANOVA $p=0.0001$ with Holm-Sidak's multiple comparisons test). Data have been normalized to control. d Percentage of differentiated $\mathrm{MBP}^{+}$ oligodendrocytes in cultures exposed for 2 days to i-EVs alone or in combination with S-FTY720-Vinylphosphonate, FTY720-Vinylphosphonate alone or S1P. Data have been normalized to control $(n=3$; Kruskal-Wallis test $p=0.0009$ with Dunn's multiple comparisons test versus control)

(2016/B/2). This study was also supported by Fondazione Cariplo grant no. 2015-0910 to MF and by Ministero dell'Istruzione, dell'Università e della Ricerca-MIUR project "Dipartimenti di Eccellenza 2018-2022" to Dept. of Pharmacological and Biomolecular Sciences and Dept. of Neuroscience Rita Levi Montalcini.

Open Access This article is distributed under the terms of the Creative Commons Attribution 4.0 International License (http://creativeco mmons.org/licenses/by/4.0/), which permits unrestricted use, distribution, and reproduction in any medium, provided you give appropriate credit to the original author(s) and the source, provide a link to the Creative Commons license, and indicate if changes were made. 


\section{References}

1. Antonucci F, Turola E, Riganti L, Caleo M, Gabrielli M, Perrotta $\mathrm{C}$ et al (2012) Microvesicles released from microglia stimulate synaptic activity via enhanced sphingolipid metabolism. EMBO J 31:1231-1240. https://doi.org/10.1038/emboj.2011.489

2. Arab T, Raffo-Romero A, Van Camp C, Lemaire Q, Le Marrec-Croq F, Drago F et al (2019) Proteomic characterisation of leech microglia extracellular vesicles (EVs): comparison between differential ultracentrifugation and Optiprep density gradient isolation. J Extracell Vesicles 8:1603048. https://doi. org/10.1080/20013078.2019.1603048

3. Baecher-Allan C, Kaskow BJ, Weiner HL (2018) Multiple sclerosis: mechanisms and immunotherapy. Neuron 97:742-768. https ://doi.org/10.1016/j.neuron.2018.01.021

4. Barenholz Y, Gibbes D, Litman BJ, Goll J, Thompson TE, Carlson RD (1977) A simple method for the preparation of homogeneous phospholipid vesicles. Biochemistry 16:2806-2810

5. Bertero A, Liska A, Pagani M, Parolisi R, Masferrer ME, Gritti $\mathrm{M}$ et al (2018) Autism-associated 16p11.2 microdeletion impairs prefrontal functional connectivity in mouse and human. Brain $\mathbf{J}$ Neurol 141:2055-2065. https://doi.org/10.1093/brain/awy111

6. Bin JM, Harris SN, Kennedy TE (2016) The oligodendrocytespecific antibody 'CC1' binds Quaking 7. J Neurochem 139:181186. https://doi.org/10.1111/jnc. 13745

7. Bisht K, Sharma K, Lacoste B, Tremblay ME (2016) Dark microglia: why are they dark? Commun Integr Biol 9:e1230575. https ://doi.org/10.1080/19420889.2016.1230575

8. Blakemore WF, Gilson JM, Crang AJ (2003) The presence of astrocytes in areas of demyelination influences remyelination following transplantation of oligodendrocyte progenitors. Exp Neurol 184:955-963. https://doi.org/10.1016/S0014-4886(03)00347 $-9$

9. Boda E, Di Maria S, Rosa P, Taylor V, Abbracchio MP, Buffo A (2015) Early phenotypic asymmetry of sister oligodendrocyte progenitor cells after mitosis and its modulation by aging and extrinsic factors. Glia 63:271-286. https://doi.org/10.1002/ glia. 22750

10. Bonavita E, Gentile S, Rubino M, Maina V, Papait R, Kunderfranco P et al (2015) PTX3 is an extrinsic oncosuppressor regulating complement-dependent inflammation in cancer. Cell 160:700-714. https://doi.org/10.1016/j.cell.2015.01.004

11. Bonfanti E, Gelosa P, Fumagalli M, Dimou L, Vigano F, Tremoli E et al (2017) The role of oligodendrocyte precursor cells expressing the GPR17 receptor in brain remodeling after stroke. Cell Death Dis 8:e2871. https://doi.org/10.1038/cddis.2017.256

12. Brambilla R (2019) The contribution of astrocytes to the neuroinflammatory response in multiple sclerosis and experimental autoimmune encephalomyelitis. Acta Neuropathol 137:757-783. https://doi.org/10.1007/s00401-019-01980-7

13. Brambilla R, Ashbaugh JJ, Magliozzi R, Dellarole A, Karmally S, Szymkowski DE et al (2011) Inhibition of soluble tumour necrosis factor is therapeutic in experimental autoimmune encephalomyelitis and promotes axon preservation and remyelination. Brain J Neurol 134:2736-2754. https://doi.org/10.1093/ brain/awr199

14. Butovsky O, Landa G, Kunis G, Ziv Y, Avidan H, Greenberg N et al (2006) Induction and blockage of oligodendrogenesis by differently activated microglia in an animal model of multiple sclerosis. J Clin Invest 116:905-915. https://doi.org/10.1172/ JCI26836

15. Casella G, Colombo F, Finardi A, Descamps H, Ill-Raga G, Spinelli A et al (2018) Extracellular vesicles containing IL-4 modulate neuroinflammation in a mouse model of multiple sclerosis. Mol Ther 26:2107-2118. https://doi.org/10.1016/j. ymthe.2018.06.024

16. Chan JR, Watkins TA, Cosgaya JM, Zhang C, Chen L, Reichardt LF et al (2004) NGF controls axonal receptivity to myelination by Schwann cells or oligodendrocytes. Neuron 43:183-191. https ://doi.org/10.1016/j.neuron.2004.06.024

17. Chang C, Lang H, Geng N, Wang J, Li N, Wang X (2013) Exosomes of BV-2 cells induced by alpha-synuclein: important mediator of neurodegeneration in PD. Neurosci Lett 548:190 195. https://doi.org/10.1016/j.neulet.2013.06.009

18. Cherry JD, Olschowka JA, O'Banion MK (2014) Neuroinflammation and M2 microglia: the good, the bad, and the inflamed. J Neuroinflamm 11:98. https://doi.org/10.1186/1742-2094-11-98

19. Choi JW, Gardell SE, Herr DR, Rivera R, Lee CW, Noguchi K et al (2011) FTY720 (fingolimod) efficacy in an animal model of multiple sclerosis requires astrocyte sphingosine 1-phosphate receptor 1 (S1P1) modulation. Proc Natl Acad Sci USA 108:751756. https://doi.org/10.1073/pnas.1014154108

20. Clarke LE, Liddelow SA, Chakraborty C, Munch AE, Heiman M, Barres BA (2018) Normal aging induces A1-like astrocyte reactivity. Proc Natl Acad Sci USA 115:E1896-E1905. https:// doi.org/10.1073/pnas.1800165115

21. Colombo E, Farina C (2016) Astrocytes: key regulators of neuroinflammation. Trends Immunol 37:608-620. https://doi. org/10.1016/j.it.2016.06.006

22. Colombo F, Bastoni M, Nigro A, Podini P, Finardi A, Casella $\mathrm{G}$ et al (2018) Cytokines stimulate the release of microvesicles from myeloid cells independently from the P2X7 receptor/acid sphingomyelinase pathway. Front Immunol 9:204. https://doi. org/10.3389/fimmu.2018.00204

23. Cui QL, Fang J, Kennedy TE, Almazan G, Antel JP (2014) Role of p38MAPK in S1P receptor-mediated differentiation of human oligodendrocyte progenitors. Glia 62:1361-1375. https://doi. org/10.1002/glia.22688

24. Domingues HS, Portugal CC, Socodato R, Relvas JB (2016) Oligodendrocyte, astrocyte, and microglia crosstalk in myelin development, damage, and repair. Front Cell Dev Biol 4:71. https ://doi.org/10.3389/fcell.2016.00071

25. Drago F, Lombardi M, Prada I, Gabrielli M, Joshi P, Cojoc D et al (2017) ATP modifies the proteome of extracellular vesicles released by microglia and influences their action on astrocytes. Front Pharmacol 8:910. https://doi.org/10.3389/fphar .2017.00910

26. Dubochet J, Adrian M, Chang JJ, Homo JC, Lepault J, McDowall AW et al (1988) Cryo-electron microscopy of vitrified specimens. Q Rev Biophys 21:129-228

27. El Hajj H, Savage JC, Bisht K, Parent M, Vallieres L, Rivest S et al (2019) Ultrastructural evidence of microglial heterogeneity in Alzheimer's disease amyloid pathology. J Neuroinflamm 16:87. https://doi.org/10.1186/s12974-019-1473-9

28. Fischer MT, Wimmer I, Hoftberger R, Gerlach S, Haider L, Zrzavy T et al (2013) Disease-specific molecular events in cortical multiple sclerosis lesions. Brain J Neurol 136:1799-1815. https://doi.org/10.1093/brain/awt110

29. Fitzgerald W, Freeman ML, Lederman MM, Vasilieva E, Romero R, Margolis L (2018) A system of cytokines encapsulated in extracellular vesicles. Sci Rep 8:8973. https://doi.org/10.1038/ s41598-018-27190-x

30. Franklin RJ, Goldman SA (2015) Glia disease and repair-remyelination. Csh Perspect Biol 7:a020594. https://doi.org/10.1101/ cshperspect.a020594

31. Fumagalli M, Bonfanti E, Daniele S, Zappelli E, Lecca D, Martini $\mathrm{C}$ et al (2015) The ubiquitin ligase Mdm2 controls oligodendrocyte maturation by intertwining mTOR with G proteincoupled receptor kinase 2 in the regulation of GPR17 receptor 
desensitization. Glia 63:2327-2339. https://doi.org/10.1002/ glia.22896

32. Fumagalli M, Daniele S, Lecca D, Lee PR, Parravicini C, Fields RD et al (2011) Phenotypic changes, signaling pathway, and functional correlates of GPR17-expressing neural precursor cells during oligodendrocyte differentiation. J Biol Chem 286:1059310604. https://doi.org/10.1074/jbc.M110.162867

33. Fumagalli M, Lecca D, Abbracchio MP (2016) CNS remyelination as a novel reparative approach to neurodegenerative diseases: the roles of purinergic signaling and the $\mathrm{P} 2 \mathrm{Y}$-like receptor GPR17. Neuropharmacol 104:82-93. https://doi.org/10.1016/j. neuropharm.2015.10.005

34. Gabrielli M, Battista N, Riganti L, Prada I, Antonucci F, Cantone L et al (2015) Active endocannabinoids are secreted on extracellular membrane vesicles. EMBO Rep 16:213-220. https://doi. org/10.15252/embr.201439668

35. Ginhoux F, Greter M, Leboeuf M, Nandi S, See P, Gokhan S et al (2010) Fate mapping analysis reveals that adult microglia derive from primitive macrophages. Science 330:841-845. https://doi.org/10.1126/science.1194637

36. Giunti D, Parodi B, Usai C, Vergani L, Casazza S, Bruzzone S et al (2012) Mesenchymal stem cells shape microglia effector functions through the release of CX3CL1. Stem cells 30:20442053. https://doi.org/10.1002/stem.1174

37. Goldmann T, Wieghofer P, Muller PF, Wolf Y, Varol D, Yona S et al (2013) A new type of microglia gene targeting shows TAK1 to be pivotal in CNS autoimmune inflammation. Nat Neurosci 16:1618-1626. https://doi.org/10.1038/nn.3531

38. Groves A, Kihara Y, Chun J (2013) Fingolimod: direct CNS effects of sphingosine 1-phosphate $(\mathrm{S} 1 \mathrm{P})$ receptor modulation and implications in multiple sclerosis therapy. J Neurol Sci 328:9-18. https://doi.org/10.1016/j.jns.2013.02.011

39. Gualerzi A, Kooijmans SAA, Niada S, Picciolini S, Brini AT, Camussi G et al (2019) Raman spectroscopy as a quick tool to assess purity of extracellular vesicle preparations and predict their functionality. J Extracell Vesicles 8:1568780. https://doi. org/10.1080/20013078.2019.1568780

40. Gualerzi A, Niada S, Giannasi C, Picciolini S, Morasso C, Vanna R et al (2017) Raman spectroscopy uncovers biochemical tissue-related features of extracellular vesicles from mesenchymal stromal cells. Sci Rep 7:9820. https://doi.org/10.1038/ s41598-017-10448-1

41. Hall SM (1972) The effect of injections of lysophosphatidyl choline into white matter of the adult mouse spinal cord. J Cell Sci 10:535-546

42. Hammond TR, Gadea A, Dupree J, Kerninon C, Nait-Oumesmar B, Aguirre A et al (2014) Astrocyte-derived endothelin-1 inhibits remyelination through notch activation. Neuron 81:588-602. https://doi.org/10.1016/j.neuron.2013.11.015

43. Harris LA, Baffy N (2017) Modulation of the gut microbiota: a focus on treatments for irritable bowel syndrome. Postgrad Med 129:872-888. https://doi.org/10.1080/00325 481.2017.1383819

44. Hinks GL, Franklin RJ (2000) Delayed changes in growth factor gene expression during slow remyelination in the CNS of aged rats. Mol Cell Neurosci 16:542-556. https://doi.org/10.1006/ mone.2000.0897

45. Hooper C, Sainz-Fuertes R, Lynham S, Hye A, Killick R, Warley A et al (2012) Wnt3a induces exosome secretion from primary cultured rat microglia. BMC Neurosci 13:144. https://doi. org/10.1186/1471-2202-13-144

46. Kalakh S, Mouihate A (2017) Androstenediol reduces demyelination-induced axonopathy in the rat corpus callosum: impact on microglial polarization. Front Cell Neurosci 11:49. https://doi. org/10.3389/fncel.2017.00049
47. Kemanetzoglou E, Andreadou E (2017) CNS Demyelination with TNF-alpha Blockers. Curr Neurol Neurosci 17:36. https://doi. org/10.1007/s11910-017-0742-1

48. Keough MB, Jensen SK, Yong VW (2015) Experimental demyelination and remyelination of murine spinal cord by focal injection of lysolecithin. JoVE. https://doi.org/10.3791/52679

49. Kimura A, Ohmori T, Kashiwakura Y, Ohkawa R, Madoiwa S, Mimuro J et al (2008) Antagonism of sphingosine 1-phosphate receptor-2 enhances migration of neural progenitor cells toward an area of brain. Stroke 39:3411-3417. https://doi.org/10.1161/ STROKEAHA.108.514612

50. Kotter MR, Setzu A, Sim FJ, Van Rooijen N, Franklin RJ (2001) Macrophage depletion impairs oligodendrocyte remyelination following lysolecithin-induced demyelination. Glia 35:204-212

51. Krafft C, Wilhelm K, Eremin A, Nestel S, von Bubnoff N, Schultze-Seemann W et al (2017) A specific spectral signature of serum and plasma-derived extracellular vesicles for cancer screening. Nanomed Nanotechnol 13:835-841. https://doi. org/10.1016/j.nano.2016.11.016

52. Kriebel PW, Majumdar R, Jenkins LM, Senoo H, Wang W, Ammu $S$ et al (2018) Extracellular vesicles direct migration by synthesizing and releasing chemotactic signals. J Cell Biol 217:2891-2910. https://doi.org/10.1083/jcb.201710170

53. Kucharova K, Stallcup WB (2017) Distinct NG2 proteoglycandependent roles of resident microglia and bone marrow-derived macrophages during myelin damage and repair. PLoS One 12:e0187530. https://doi.org/10.1371/journal.pone.0187530

54. Kuhlmann T, Miron V, Cui Q, Wegner C, Antel J, Bruck W (2008) Differentiation block of oligodendroglial progenitor cells as a cause for remyelination failure in chronic multiple sclerosis. Brain 131:1749-1758. https://doi.org/10.1093/brain/awn096

55. Liddelow SA, Guttenplan KA, Clarke LE, Bennett FC, Bohlen CJ, Schirmer L et al (2017) Neurotoxic reactive astrocytes are induced by activated microglia. Nature 541:481-487. https://doi. org/10.1038/nature21029

56. Livak KJ, Schmittgen TD (2001) Analysis of relative gene expression data using real-time quantitative PCR and the 2(-Delta Delta C(T)). Methods 25:402-408. https://doi.org/10.1006/ meth.2001.1262

57. Locatelli G, Theodorou D, Kendirli A, Jordao MJC, Staszewski O, Phulphagar K et al (2018) Mononuclear phagocytes locally specify and adapt their phenotype in a multiple sclerosis model. Nat Neurosci 21:1196-1208. https://doi.org/10.1038/s4159 3-018-0212-3

58. Lock C, Oksenberg J, Steinman L (1999) The role of TNFalpha and lymphotoxin in demyelinating disease. Ann Rheum Dis 58(Suppl 1):I121-I128

59. Maimone D, Gregory S, Arnason BG, Reder AT (1991) Cytokine levels in the cerebrospinal fluid and serum of patients with multiple sclerosis. J Neuroimmunol 32:67-74

60. Mallucci G, Peruzzotti-Jametti L, Bernstock JD, Pluchino S (2015) The role of immune cells, glia and neurons in white and gray matter pathology in multiple sclerosis. Prog Neurobiol 127-128:1-22. https://doi.org/10.1016/j.pneurobio.2015.02.003

61. Marrone MC, Morabito A, Giustizieri M, Chiurchiu V, Leuti A, Mattioli M et al (2017) TRPV1 channels are critical brain inflammation detectors and neuropathic pain biomarkers in mice. Nature Commun 8:15292. https://doi.org/10.1038/ncomms 1529 2

62. Mayo L, Trauger SA, Blain M, Nadeau M, Patel B, Alvarez JI et al (2014) Regulation of astrocyte activation by glycolipids drives chronic CNS inflammation. Nat Med 20:1147-1156. https ://doi.org/10.1038/nm.3681

63. Miron VE (2017) Microglia-driven regulation of oligodendrocyte lineage cells, myelination, and remyelination. J Leukoc Biol 101:1103-1108. https://doi.org/10.1189/jlb.3RI1116-494R 
64. Miron VE, Boyd A, Zhao JW, Yuen TJ, Ruckh JM, Shadrach JL et al (2013) M2 microglia and macrophages drive oligodendrocyte differentiation during CNS remyelination. Nat Neurosci 16:1211-1218. https://doi.org/10.1038/nn.3469

65. Miron VE, Ludwin SK, Darlington PJ, Jarjour AA, Soliven B, Kennedy TE et al (2010) Fingolimod (FTY720) enhances remyelination following demyelination of organotypic cerebellar slices. Am J Pathol 176:2682-2694. https://doi.org/10.2353/ajpat h.2010.091234

66. Mycko MP, Papoian R, Boschert U, Raine CS, Selmaj KW (2003) cDNA microarray analysis in multiple sclerosis lesions: detection of genes associated with disease activity. Brain 126:1048-1057

67. Nash B, Thomson CE, Linington C, Arthur AT, McClure JD, McBride MW et al (2011) Functional duality of astrocytes in myelination. J Neurosci 31:13028-13038. https://doi. org/10.1523/JNEUROSCI.1449-11.2011

68. Okabe M, Ikawa M, Kominami K, Nakanishi T, Nishimune Y (1997) 'Green mice' as a source of ubiquitous green cells. FEBS Lett 407:313-319

69. Olah M, Amor S, Brouwer N, Vinet J, Eggen B, Biber K et al (2012) Identification of a microglia phenotype supportive of remyelination. Glia 60:306-321. https://doi.org/10.1002/ glia.21266

70. Orlov I, Myasnikov AG, Andronov L, Natchiar SK, Khatter H, Beinsteiner B et al (2017) The integrative role of cryo electron microscopy in molecular and cellular structural biology. Biol Cell 109:81-93. https://doi.org/10.1111/boc.201600042

71. Paolicelli RC, Bergamini G, Rajendran L (2018) Cell-to-cell communication by extracellular vesicles: focus on microglia. Neuroscience. https://doi.org/10.1016/j.neuroscien ce.2018.04.003

72. Peferoen L, Kipp M, van der Valk P, van Noort JM, Amor S (2014) Oligodendrocyte-microglia cross-talk in the central nervous system. Immunology 141:302-313. https://doi.org/10.1111/ imm. 12163

73. Prada I, Amin L, Furlan R, Legname G, Verderio C, Cojoc D (2016) A new approach to follow a single extracellular vesiclecell interaction using optical tweezers. Biotechniques 60:35-41. https://doi.org/10.2144/000114371

74. Prada I, Furlan R, Matteoli M, Verderio C (2013) Classical and unconventional pathways of vesicular release in microglia. Glia 61:1003-1017. https://doi.org/10.1002/glia.22497

75. Prada I, Gabrielli M, Turola E, Iorio A, D’Arrigo G, Parolisi R et al (2018) Glia-to-neuron transfer of miRNAs via extracellular vesicles: a new mechanism underlying inflammation-induced synaptic alterations. Acta Neuropathol 135:529-550. https:// doi.org/10.1007/s00401-017-1803-x

76. Pusic AD, Kraig RP (2014) Youth and environmental enrichment generate serum exosomes containing miR-219 that promote CNS myelination. Glia 62:284-299. https://doi.org/10.1002/ glia.22606

77. Pusic KM, Pusic AD, Kraig RP (2016) Environmental enrichment stimulates immune cell secretion of exosomes that promote CNS myelination and may regulate inflammation. Cell Mol Neurobiol 36:313-325. https://doi.org/10.1007/s10571-015-0269-4

78. Quigley EMM (2017) Microbiota-brain-gut axis and neurodegenerative diseases. Curr Neurol Neurosci 17:94. https://doi. org/10.1007/s11910-017-0802-6

79. Riboni L, Viani P, Bassi R, Giussani P, Tettamanti G (2000) Cultured granule cells and astrocytes from cerebellum differ in metabolizing sphingosine. J Neurochem 75:503-510

80. Riboni L, Viani P, Tettamanti G (2000) Estimating sphingolipid metabolism and trafficking in cultured cells using radiolabeled compounds. Method Enzymol 311:656-682

81. Rothhammer V, Kenison JE, Tjon E, Takenaka MC, de Lima KA, Borucki DM et al (2017) Sphingosine 1-phosphate receptor modulation suppresses pathogenic astrocyte activation and chronic progressive CNS inflammation. Proc Natl Acad Sci USA 114:2012-2017. https://doi.org/10.1073/pnas.1615413114

82. Ruckh JM, Zhao JW, Shadrach JL, van Wijngaarden P, Rao TN, Wagers AJ et al (2012) Rejuvenation of regeneration in the aging central nervous system. Cell Stem Cell 10:96-103. https://doi. org/10.1016/j.stem.2011.11.019

83. Shields S, Gilson J, Blakemore W, Franklin R (2000) Remyelination occurs as extensively but more slowly in old rats compared to young rats following fliotoxin-induced CNS demyelination. Glia 29:102

84. Sim FJ, Zhao C, Penderis J, Franklin RJ (2002) The age-related decrease in CNS remyelination efficiency is attributable to an impairment of both oligodendrocyte progenitor recruitment and differentiation. J Neurosci 22:2451-2459. https://doi. org/10.1523/JNEUROSCI.22-07-02451.2002

85. Smith ZJ, Lee C, Rojalin T, Carney RP, Hazari S, Knudson A et al (2015) Single exosome study reveals subpopulations distributed among cell lines with variability related to membrane content. J Extracell Vesicles 4:28533. https://doi.org/10.3402/ jev.v4.28533

86. Soni S, O'Dea KP, Tan YY, Cho K, Abe E, Romano R et al (2019) ATP redirects cytokine trafficking and promotes novel membrane TNF signaling via microvesicles. FASEB J. https:// doi.org/10.1096/fj.201802386r

87. Sun D, Yu Z, Fang X, Liu M, Pu Y, Shao Q et al (2017) LncRNA GAS5 inhibits microglial M2 polarization and exacerbates demyelination. EMBO Rep 18:1801-1816. https://doi.org/10.15252/ embr.201643668

88. Tatischeff I, Larquet E, Falcon-Perez JM, Turpin PY, Kruglik SG (2012) Fast characterisation of cell-derived extracellular vesicles by nanoparticles tracking analysis, cryo-electron microscopy, and Raman tweezers microspectroscopy. J Extracell Vesicles. https:// doi.org/10.3402/jev.v1i0.19179

89. Tremblay ME, Lowery RL, Majewska AK (2010) Microglial interactions with synapses are modulated by visual experience. PLoS Biol 8:e1000527. https://doi.org/10.1371/journ al.pbio. 1000527

90. Turola E, Furlan R, Bianco F, Matteoli M, Verderio C (2012) Microglial microvesicle secretion and intercellular signaling. Front Physiol 3:149. https://doi.org/10.3389/fphys.2012.00149

91. Valentin-Torres A, Savarin C, Hinton DR, Phares TW, Bergmann CC, Stohlman SA (2016) Sustained TNF production by central nervous system infiltrating macrophages promotes progressive autoimmune encephalomyelitis. J Neuroinflamm 13:46. https:// doi.org/10.1186/s12974-016-0513-y

92. Valentine WJ, Kiss GN, Liu J, Shuyu E, Gotoh M, MurakamiMurofushi K, Pham TC et al (2010) (S)-FTY720-vinylphosphonate, an analogue of the immunosuppressive agent FTY720, is a pan-antagonist of sphingosine 1-phosphate GPCR signaling and inhibits autotaxin activity. Cell Signal 22:1543-1553. https://doi. org/10.1016/j.cellsig.2010.05.023

93. Van Oosten BW, Barkhof F, Scholten PE, von Blomberg BM, Ader HJ, Polman CH (1998) Increased production of tumor necrosis factor alpha, and not of interferon gamma, preceding disease activity in patients with multiple sclerosis. Arch Neurol 55:793-798

94. Verderio C, Gabrielli M, Giussani P (2018) Role of sphingolipids in the biogenesis and biological activity of extracellular vesicles. J Lipid Res 59:1325-1340. https://doi.org/10.1194/jlr.R083915

95. Verderio C, Muzio L, Turola E, Bergami A, Novellino L, Ruffini $F$ et al (2012) Myeloid microvesicles are a marker and therapeutic target for neuroinflammation. Ann Neurol 72:610-624. https ://doi.org/10.1002/ana.23627

96. Viani P, Giussani P, Brioschi L, Bassi R, Anelli V, Tettamanti $\mathrm{G}$ et al (2003) Ceramide in nitric oxide inhibition of glioma cell 
growth. Evidence for the involvement of ceramide traffic. J Biol Chem 278:9592-9601. https://doi.org/10.1074/jbc.M207729200

97. Voss EV, Skuljec J, Gudi V, Skripuletz T, Pul R, Trebst C et al (2012) Characterisation of microglia during de- and remyelination: can they create a repair promoting environment? Neurobiol Dis 45:519-528. https://doi.org/10.1016/j.nbd.2011.09.008

98. Yang Y, Boza-Serrano A, Dunning CJR, Clausen BH, Lambertsen KL, Deierborg T (2018) Inflammation leads to distinct populations of extracellular vesicles from microglia. J Neuroinflamm 15:168. https://doi.org/10.1186/s12974-018-1204-7

99. Yun SP, Kam TI, Panicker N, Kim S, Oh Y, Park JS et al (2018) Block of A1 astrocyte conversion by microglia is neuroprotective in models of Parkinson's disease. Nat Med 24:931-938. https:// doi.org/10.1038/s41591-018-0051-5

100. Zabala A, Vazquez-Villoldo N, Rissiek B, Gejo J, Martin A, Palomino A et al (2018) P2X4 receptor controls microglia activation and favors remyelination in autoimmune encephalitis. EMBO Mol Med. https://doi.org/10.15252/emmm.201708743
101. Zanier ER, Pischiutta F, Riganti L, Marchesi F, Turola E, Fumagalli $S$ et al (2014) Bone marrow mesenchymal stromal cells drive protective M2 microglia polarization after brain trauma. Neurotherapeutics 11:679-695. https://doi.org/10.1007/s1331 1-014-0277-y

102. Zappia E, Casazza S, Pedemonte E, Benvenuto F, Bonanni I, Gerdoni E et al (2005) Mesenchymal stem cells ameliorate experimental autoimmune encephalomyelitis inducing T-cell anergy. Blood 106:1755-1761. https://doi.org/10.1182/blood $-2005-04-1496$

Publisher's Note Springer Nature remains neutral with regard to jurisdictional claims in published maps and institutional affiliations.

\section{Affiliations}

Marta Lombardi ${ }^{1,2} \cdot$ Roberta Parolisi $^{3} \cdot$ Federica Scaroni $^{2} \cdot$ Elisabetta Bonfanti ${ }^{4} \cdot$ Alice Gualerzi $^{5}$.

Martina Gabrielli ${ }^{2}$. Nicole Kerlero de Rosbo ${ }^{6}$. Antonio Uccelli ${ }^{6,7}$. Paola Giussani ${ }^{8}$. Paola Viani ${ }^{8}$. Cecilia Garlanda ${ }^{9}$. Maria P. Abbracchio ${ }^{4} \cdot$ Linda Chaabane $^{10} \cdot$ Annalisa Buffo $^{3} \cdot$ Marta Fumagalli $^{4} \cdot$ Claudia Verderio $^{2}$

1 IRCCS Humanitas, via Manzoni 56, 20089 Rozzano, Italy

2 CNR Institute of Neuroscience, via Vanvitelli 32, 20129 Milan, Italy

3 Department of Neuroscience Rita Levi-Montalcini and Neuroscience Institute Cavalieri Ottolenghi, University of Turin, Regione Gonzole 10, 10043 Orbassano, Italy

4 Department of Excellence: Department of Pharmacological and Biomolecular Sciences (DiSFeB), University of Milan, via Balzaretti 9, 20133 Milan, Italy

5 IRCCS Fondazione Don Carlo Gnocchi, via Capecelatro 66, 20148 Milan, Italy

6 Department of Neurology, Rehabilitation, Ophthalmology, Genetics, Maternal and Child Health (DINOGMI),
University of Genoa, Largo Paolo Daneo 3, 16132 Genoa, Italy

7 IRCCS Ospedale Policlinico San Martino, Largo Rosanna Benzi 10, 16132 Genoa, Italy

8 Department of Biotechnology and Translational Medicine, University of Milan, 20090 Segrate, Italy

9 Humanitas University, via Manzoni 56, 20089 Rozzano, Italy

10 Institute of Experimental Neurology (INSPE), San Raffaele Scientific Institute, Via Olgettina Milano 58, 20132 Milan, Italy 